Pacific Journal of Mathematics

HARMONIC ANALYSIS ON EXPONENTIAL SOLVABLE
HOMOGENEOUS SPACES: THE ALGEBRAS OR SYMMETRIC
CASES

RONALD LESLIE LIPSMAN 


\title{
HARMONIC ANALYSIS ON \\ EXPONENTIAL SOLVABLE HOMOGENEOUS SPACES: THE ALGEBRAIC OR SYMMETRIC CASES
}

\author{
RONALD L. LIPSMAN
}

\begin{abstract}
One of the main results of this paper is a complete description of the spectral decomposition of the quasi-regular representation of an arbitrary exponential solvable symmetric space. Benoist had shown previously that such a representation is multiplicity-free, but he was unable to compute the precise spectrum and spectral measure. More generally, the quasi-regular representation is considered for any exponential solvable homogeneous space. In previous work of the author and Messrs. Corwin, Greenleaf and Grélaud, the analysis of these representations was carried out in the nilpotent case. The spectral decomposition arrived at was in terms of the Kirillov orbital parameters. Corresponding results are obtained here for algebraic exponential solvable homogeneous spaces in case the stability subgroup is either: a Levi component, or its nilradical is multiplicity-free in the nilradical of the homogeneous group. The description of the spectral decomposition in the Mackey parameters is also obtained for these representations.
\end{abstract}

1. Introduction. The themes developed in this paper have their origin in the subject matter of [2], [3], [4], [16], [17], [21]. Namely, we study harmonic analysis on homogeneous spaces $G / H$, where both $G$ and $H$ are connected Lie groups. The basic problems considered in the above papers concern the spectral analysis of the unitary representations

$$
\operatorname{Ind}_{H}^{G} \nu \text { and }\left.\pi\right|_{H},
$$

where $\pi$ and $\nu$ are irreducible unitary representations of $G$ and $H$ respectively. In this paper we shall restrict attention to the induced representation with $\nu=1$-what is usually called the quasi-regular representation of $G$ on $L^{2}(G / H)$. Moreover, we shall be primarily concerned with the cases: $G / H$ symmetric (with $G$ non-semisimple), or $G$ exponential solvable, or $G$ algebraic.

The spectral analysis of the quasi-regular representation means a direct integral decomposition of $\operatorname{Ind}_{H}^{G} 1$ into irreducible constituents. In particular it calls for an explicit parameterization of the spectrum, the spectral multiplicities and the spectral measure. Since we are dealing 
with non-semisimple groups, there are only two possible candidates for the parameters: Mackey Machine parameters or Kirillov orbital parameters. Mackey parameters will usually suffice to describe any particular example. But it is to the orbital parameters that we look for general descriptions of harmonic analysis on non-semisimple homogeneous spaces. As one knows from previous efforts in non-semisimple groups [1], [17], in order to obtain the final form of the spectral decomposition in orbital parameters, one must derive and employ the Mackey parameters as an intermediate tool.

It is our goal to give an orbital description of the spectral decomposition for the quasi-regular representation of an arbitrary exponential solvable homogeneous space. There are two approaches one can take towards achieving that goal. One approach is to try to generalize the method used in [3] or [17]. This would require a long and complicated argument involving mathematical induction and structure theory. The nilpotent structure causes the argument in [17] to split into four different special subcases. It is apparent that a corresponding argument for arbitrary exponential solvable homogeneous spaces would require more than a dozen such special cases. A conceptually prettier approach is the following: first, utilize the fact that the nilpotent case is done; then extend it to algebraic exponential solvable groups by the three-step procedure of [15] (that is treat the case of abelian unipotent radical, then Heisenberg unipotent radical and finally general unipotent radical); and then proceed to arbitrary groups à la Pukanszky (see [19]). It is the latter approach we adopt here. Although we don't succeed completely, we do obtain the explicit spectral decomposition in orbital parameters for the quasi-regular representation when $H$ is a Levi component (Thm. 4.1), or when its unipotent radical is multiplicity-free ( $\mathrm{Thm}$. 5.2). We also obtain it for arbitrary exponential solvable symmetric spaces (Thm. 6.2 ), thereby settling a conjecture of Benoist.

The arrangement of the paper is as follows. In $\S 2$ we describe the basic goal-i.e. precisely what the orbital spectrum formula should look like for exponential solvable homogeneous spaces $G / H$ (Def. 2.1). We show the formula is true for the pair $(G H)$ if it is true for a pair $(N, H)$ with $H \subset N \subset G, N$ normal of co-dimension 1 in $G$ (Theorem 2.2). We then deduce that for algebraic groups $G=A N$, it is enough to consider subgroups of the form $H=A M, M \subset N$ (Lemma 2.4). In $\S 3$, we examine the Weil representations (that arise from the Mackey Machine) as representations of the split abelian groups $A_{\gamma}, \gamma \in \hat{N}$. We 
compute their spectrum (Prop. 3.4). We also give a general result on their spectrum when $A_{\gamma}$ is only reductive (Thm. 3.2). In $\S 4$, we give the orbital spectrum formula for co-normal subgroups (i.e. $M$ trivial) (Thm. 4.2). This uses the Mackey parameterization of co-normally induced representations (derived in [17, Thm. 7.1]). This Mackey parameterization is generalized in $\S 5$ to $L^{2}(A N / A M)$ (Thm. 5.1), and then used to obtain the orbital spectrum formula when $L^{2}(N / M)$ is multiplicity-free (Thm. 5.2). Finally, these results are employed in $\S 6$ to settle Benoist's conjecture-namely we obtain the orbital spectrum formula for arbitrary exponential solvable symmetric spaces (Thm. $6.2)$.

1a. Notation. In this paper $G$ exponential solvable means $G$ is simply connected and its Lie algebra $\mathfrak{g}$ is solvable and has no purely imaginary eigenvalues. $G$ algebraic means that $G$ is the group of real points of a complex algebraic group $\mathbf{G}$ defined over $\mathbb{R}$. If $\mathbf{G}=\mathbf{A N}$ is a Levi decomposition, then $G=A N$, where $N=\mathbf{N} \cap G$ (referred to as the unipotent radical) is characteristic, simply connected and nilpotent, and $A=\mathbf{A} \cap G$ (referred to as a Levi component) is a reductive group acting semisimply on $\mathfrak{n}$. When $G^{0}$ is exponential, $A^{0}$ is a vector group. In that case we shall write $G$ (resp. $A$ ) for $G^{0}$ (resp. $A^{0}$ ) and refer to $G=A N$ as an algebraic exponential solvable group. For $G$ exponential solvable, $\hat{G}$ is in bijective correspondence with $\mathfrak{g}^{*} / G$, the set of co-adjoint orbits. The corresponding terminology is: $\pi \in \hat{G} \hookrightarrow \mathscr{O}_{\pi} \in \mathfrak{g}^{*} / G ; \varphi \in \mathfrak{g}^{*}$ or $\Omega \in \mathfrak{g}^{*} / G \hookrightarrow \pi_{\varphi}$ or $\pi_{\Omega}$. For any $\varphi \in \mathfrak{g}^{*}$, we can find $\mathfrak{b} \subset \mathfrak{g}$, a maximal totally isotropic subalgebra (for the skew form $B_{\varphi}(X, Y)=\varphi[X, Y]$ ) which satisfies the Pukanszky condition. The character $\chi_{\varphi}: \exp X \rightarrow e^{i \varphi(x)}, X \in \mathfrak{b}$, is well-defined and $\pi_{\varphi}=\operatorname{Ind}_{B}^{G} \chi_{\varphi}$.

The symbol $\rho_{G}$ shall denote the regular representation of $G, \rho_{G, H}$ stands for the quasi-regular representation $\operatorname{Ind}_{H}^{G} 1$. If $\nu$ is a unitary representation of $H$ in the Hilbert space $\mathscr{V}$, we set

$$
\begin{aligned}
& L^{2}(G ; H ; \nu)=\{f: G \rightarrow \mathscr{V} \text { measurable, } \\
& \left.f(h g)=\nu(h) f(g), h \in H, g \in G, \int_{G / H}\|f\|^{2}<\infty\right\},
\end{aligned}
$$

it being understood that a quasi-invariant measure on $G / H$ has been fixed beforehand. If $\nu=1$, we write $L^{2}(G ; H)$ for $L^{2}(G ; H ; 1)$. Finally, if $X$ is a locally compact space with Borel measure $\mu$, and $A$ is a group 
of Borel isomorphisms of $X$ which leave $\mu$ relatively invariant, we write $\delta_{X}(A)$ for the modulus

$$
\delta_{X}(a) \int_{X} f\left(a^{-1} \cdot x\right) d \mu(x)=\int_{X} f(x) d \mu(x) .
$$

If $X$ is a group $G$ and $A=G$ acts by inner automorphism, $\delta_{G}$ gives the modular function. If $X=G / H$, we shall write $\delta_{G, H}$ in place of $\delta_{G / H}$. If $A=H$, then $\delta_{G, H}$ agrees with the quotient of the modular functions (see [10]).

2. Orbital spectrum for subnormal homogeneous spaces. Our prime interest is to describe the orbital spectrum for $\operatorname{Ind}_{H}^{G} 1$, or more generally $\operatorname{Ind}_{H}^{G} \nu$, when $G$ is exponential solvable and $H$ is a connected subgroup. This is carried out completely in [17] if $G$ is nilpotent or if $H$ is normal. The normal case is treated by combining the Kirillov-Bernat orbital parameters with the Mackey parameters as derived in [17]. When $G$ is nilpotent, the proof uses induction on $\operatorname{dim} \mathrm{G} / \mathrm{H}$-a key point being that for $G$ nilpotent one can always place a normal subgroup between $H$ and $G$-and then it employs the normal case in co-dimension one. It is natural to ask how far this scenario can be pushed when $G$ is only exponential solvable. Of course, between any $H$ and $G$ there may not lie any normal subgroups. But the important point I wish to make is that, if an intermediate normal subgroup does exist, then basically the same reasoning as in [17] can be carried through. We now make that precise.

Definition 2.1. Let $G$ be exponential solvable, $H \subset G$ a connected subgroup. (i) We say that $H$ is subnormal if there is a connected proper normal subgroup $N$ of $G$ which contains $H$. We say $H$ is strongly subnormal if $N$ can be found of codimension 1. (ii) For $\nu \in \hat{H}$ we say that $\operatorname{Ind}_{H}^{G} \nu$ obeys the orbital spectrum formula if the result [17, Thm. 3.1] is valid, i.e.

$$
\operatorname{Ind}_{H}^{G} \nu=\int_{p^{-1}\left(\mathscr{O}_{\nu}\right) / H}^{\oplus} \pi_{\varphi} d \mu_{G, H}^{\nu}(\varphi),
$$

$\mu_{G, H}^{\nu}$ the push-forward of the natural measure on $p^{-1}\left(\mathscr{O}_{\nu}\right)$ under the canonical projection $p: \mathfrak{g}^{*} \rightarrow \mathfrak{h}^{*}$.

It follows from the work of [17, Thm. 3.5] that when $\operatorname{Ind}_{H}^{G} \nu$ obeys the orbital spectrum formula, we also have the multiplicity formula

$$
\operatorname{Ind}_{H}^{G} \nu=\int_{G \cdot p^{-1}\left(\mathscr{O}_{\nu}\right) / G}^{\oplus} n_{\varphi}^{\nu} \pi_{\varphi} d \tilde{\mu}_{G, H}^{\nu}(\varphi)
$$


where $\tilde{\mu}_{G, H}^{\nu}$ is again the push-forward of the natural measure, and $n_{\varphi}^{\nu}=\# H$-orbits on $G \cdot \varphi \cap p^{-1}\left(\mathscr{O}_{\nu}\right)$. (The dimension criterion for finite multiplicity in [17, §1] does not hold-see [17, Expl. 8 (ii)].) Now we have

THEOREM 2.2. Let $G$ be exponential solvable, $H \subset G$ strongly subnormal, $\nu \in \hat{H}$. Let $N$ be a co-dimension 1 normal subgroup of $G$ containing $H$. If the representation $\operatorname{Ind}_{H}^{N} \nu$ obeys the orbital spectrum formula, then so does the induced representation $\operatorname{Ind}_{H}^{G} \nu$.

Proof. To prove the result we must return to [17] and examine where, beyond the existence of intermediate normal subgroups, the nilpotency of $G$ is used. In fact, the first result [17, Thm. 0.1] is true as it stands for $G$ exponential solvable and $N$ normal of co-dimension 1. Theorem 0.1 is a summary of results first stated in [9] for nilpotent groups-but it has long been known that nilpotence is not necessary. Of course co-dimension 1 normal subgroups may not exist, but if they are postulated (as in Thm. 2.2), then [17, Thm. 0.1] is valid. (Actually, the proof is not too difficult-the ideas in the proof of [17, Thm. 6.1] are all that is required.) This proves Theorem 2.2 when $\operatorname{dim} G / H=1$ or $H=N$.

Next assume $\operatorname{dim} G / H>1$ and $H \subset N \triangleleft G, \operatorname{dim} G / N=1$. We examine the argument of $[17, \S \S 2,3]$. In $\S 2$ the case that $\nu$ is a character is treated. We used nilpotence there to assert that group actions were smooth-i.e. the quotient spaces are countably separated. The same is always true of exponential solvable actions. Then we observe that in the rest of the proof in $\S 2$, nilpotence is never invoked. $G$ might as well be exponential solvable throughout the entire section. (And in the appendix as well.) In fact, the only consequence of replacing nilpotent by exponential solvable is that in case (a) the multiplicities may not be finite. The bijections constructed in the section are still intact. In $\S 3$, the proof is completed by allowing $\nu$ to be an arbitrary irreducible rather a character. Nilpotence is invoked to know that any real polarization for $\nu$ satisfies the Pukanszky condition. That is untrue for exponential solvable groups. Nevertheless, any $\nu \in \hat{H}$ can be realized by induction via some real polarization which satisfies the Pukanszky condition-i.e. $\nu=\operatorname{Ind}_{K}^{H} \chi_{\psi}, \psi \in \mathscr{O}_{\nu}, \mathfrak{k}$ a real polarization for $\psi$ such that $K \cdot \psi=\psi+\mathfrak{k}^{\perp}$. The bijection of Proposition 3.2, which comes from [3], is still legitimate. Nowhere else in $\S \S 2,3$ is nilpotence invoked or required, and so Theorem 2.2 is true. 
Now suppose $H \subset G$ and both are algebraic exponential solvable. Let $N$ be the unipotent radical of $G$.

Corollary 2.3. For any $\nu \in \hat{H}$, the induced representation $\operatorname{Ind}_{H}^{G} \nu$ obeys the orbital spectrum formula if the representation $\operatorname{Ind}_{H}^{H N} \nu$ obeys it.

Proof. The result is an immediate consequence of Theorem 2.2 if $\operatorname{dim} G / H N \leq 1$. Otherwise, the result still follows from the theorem by a simple (mathematical) induction argument. This is because $G / N$ is a vector group.

Continuing with $H \subset G$ both algebraic exponential solvable, $N$ the unipotent radical of $G$, we have

LEMMA 2.4. To prove the orbital spectrum formula for $\operatorname{Ind}_{H}^{G} \nu$ it is enough to assume there is a Levi decomposition of $\mathrm{H}$ of the form $\mathrm{H}=$ $A M$, where $M \subset N$ and $A$ is a Levi subgroup of $G$.

Proof. Consider $G_{1}=H N$, a closed normal subgroup of $G$. The only way $G=G_{1}$ can occur is if some Levi subgroup of $H$ is already a Levi subgroup of $G$. Let $A \subset H$ be such a Levi subgroup. Then if $M$ is the unipotent radical of $H$ it must be that $M \subset N$ and $H=$ $A M \subset G=A N$. Now suppose $G \neq G_{1}$. Clearly the unipotent radical of $G_{1}$ is $N$. Then if the orbital spectrum formula is proven for the pair $\left(G_{1}, H\right)$, it follows by Corollary 2.3 that it is also proven for the pair $(G, H)$. This completes the proof of the lemma.

Combining Corollary 2.3 and Lemma 2.4 we see that, for algebraic exponential solvable homogeneous spaces, to prove the orbital spectrum formula (when $\nu=1$ ) we need to consider only the induced representations

$$
\operatorname{Ind}_{A M}^{A N} 1 .
$$

The most important and most tractable example of these induced representations occurs when $M$ is trivial, that is co-normally induced representations

$$
\operatorname{Ind}_{A}^{A N} 1 \text {. }
$$

We take these up in $\S 4$ and (part of) the more general situation in $\S 5$.

3. Spectrum of the Weil representations. We have explained in $\S 2$ why co-normal induction plays a critical role in our approach to algebraic exponential solvable homogeneous spaces. The Mackey parameter description of a co-normally induced representation was obtained in [17]. Let us recall it. 
Suppose $G=H N$ is a semidirect product with $N$ normal and type I. Let $\gamma \in \hat{N}, H_{\gamma}$ the stability group, $\tilde{\gamma}$ an extension of $\gamma$ to $H_{\gamma}$ satisfying

$$
\tilde{\gamma}(h) \gamma\left(h^{-1} n h\right)=\gamma(n) \tilde{\gamma}(h), \quad n \in N, h \in H_{\gamma}
$$

(these are the Weil representations). $\tilde{\gamma}$ is specified up to a character, but the Mackey co-cycle (class) $\omega_{\gamma}$ defined by

$$
\tilde{\gamma}\left(h_{1}\right) \tilde{\gamma}\left(h_{2}\right)=\omega_{\gamma}\left(h_{1}, h_{2}\right) \tilde{\gamma}\left(h_{1} h_{2}\right), \quad h_{j} \in H_{\gamma}
$$

is uniquely determined. The Mackey Machine representations are

$$
\pi_{\gamma, \sigma}=\operatorname{Ind}_{H_{\gamma} N}^{G} \sigma \otimes \tilde{\gamma} \times \gamma, \quad \sigma \in \hat{H}_{\gamma}^{\bar{\omega}_{\gamma}} .
$$

Theorem 3.1 ([17, Thm. 7.11]). Suppose $N$ is unimodular, $\hat{N} / H$ is countably separated and $\tilde{\gamma}$ is type I for a.a. $\gamma \in \hat{N}$. Let

$$
\tilde{\gamma}=\int_{\hat{H}_{\gamma}^{\omega_{\gamma}}}^{\oplus} n_{\gamma}(\sigma) \sigma d \mu_{\gamma}(\sigma)
$$

be the unique direct integral decomposition of $\tilde{\gamma}$. Then

$$
\operatorname{Ind}_{H}^{G} 1=\int_{\hat{N} / H}^{\oplus} \int_{\hat{H}_{\gamma}^{\bar{\omega}_{\gamma}}}^{\oplus} n_{\gamma}(\bar{\sigma}) \pi_{\gamma, \sigma} d \mu_{\gamma}(\bar{\sigma}) d \dot{\mu}_{N}(\gamma),
$$

where $\dot{\mu}_{N}$ is the push-forward of the Plancherel measure $\mu_{N}$ on $\hat{N}$.

Our goal is to reconcile the Mackey parameters in Theorem 3.1 with orbital parameters for co-normally induced representations which arise in algebraic exponential solvable groups. To achieve that it is clearly important to have a good understanding of the spectrum of $\tilde{\gamma}$. In the algebraic exponential solvable situation $H$ will be split abelian. Before specializing to that case, we present a general result which may be of independent interest.

Theorem 3.2. Suppose $G$ is algebraic with Levi decomposition $G=$ $H N$. Let $\gamma \in \hat{N}$ and suppose $H_{\gamma}$ is reductive. Then

(i) there exists $\theta \in \mathscr{O}_{\gamma} \subset \mathfrak{n}^{*}$ such that $H_{\gamma}=H_{\theta}$. Hence $G_{\theta}=H_{\theta} N_{\theta}$ and there is a natural map $H_{\gamma} \rightarrow \mathrm{Sp}\left(\mathfrak{n} / \mathfrak{n}_{\theta}\right)$.

(ii) Suppose the image is of a full rank-i.e. it contains a Cartan subgroup. Then $\tilde{\gamma}$ is of finite multiplicity. If the image contains a compact Cartan subgroup, then $\tilde{\gamma}$ is multiplicity-free.

Proof. The proof of (i) may be found in [15, Lem. 3.3] where it is called the Alignment Lemma. We demonstrate part (ii). First we 
observe that if $H_{1}$ is a closed subgroup of $H$ so that $\left(H_{1}\right)_{\gamma} \subset H_{\gamma}$, then clearly

$$
\tilde{\gamma}_{H_{1}}=\tilde{\gamma}_{H_{H_{1}}} .
$$

Therefore if $\tilde{\gamma}_{H_{1}}$ is of finite multiplicity, or respectively multiplicityfree, then a fortiori $\tilde{\gamma}_{H}$ must have the same property. We combine this observation with the fact that the image of $H_{\gamma}$ inside $\operatorname{Sp}\left(\mathfrak{n} / \mathfrak{n}_{\theta}\right)$ must be closed and reductive. Hence it suffices to prove the following

LEMMA 3.3. Let $N$ be simply connected nilpotent, $\theta \in \mathfrak{n}^{*}, C$ a Cartan subgroup of $\operatorname{Sp}\left(\mathfrak{n} / \mathfrak{n}_{\theta}\right)$. Let $H$ be a group of automorphisms of $N$ fixing $\theta$ so that the corresponding homomorphism $H \rightarrow \operatorname{Sp}\left(\mathfrak{n} / \mathfrak{n}_{\theta}\right)$ has image $C$. $H$ fixes the class of $\gamma=\gamma_{\theta}$, so let $\tilde{\gamma}$ be an associated Weil representation of $H$. Then $\tilde{\gamma}$ is of uniform finite multiplicity $2^{r}$, where $r$ is the split rank of $C$. In particular, if $C$ is compact, $\tilde{\gamma}$ is multiplicity-free.

Proof. Before beginning the argument, let us note that the "degenerate" case in which $\gamma$ is a unitary character obeys the Lemma and the Theorem. In that case $\mathfrak{n}=\mathfrak{n}_{\theta}$ and $\tilde{\gamma}$ is the trivial homomorphism. If $\gamma$ is not a character, we reason as follows. We know (by [1] or [15]) that there is an $H$-invariant positive polarization $\mathfrak{m} \subset \mathfrak{n}_{c}$ for $\theta$. Let $\mathfrak{d}=\mathfrak{m} \cap \mathfrak{n}, \mathfrak{e}=(\mathfrak{m}+\overline{\mathfrak{m}}) \cap \mathfrak{n}$. Then we may realize the representation $\gamma$ as the holomorphically induced representation via $\mathfrak{m}$ from $\chi_{\theta}$ on $N_{\theta}$. Furthermore, we may take the Hilbert space for that representation to be

$$
L^{2}(\mathfrak{n} / \mathfrak{e}) \otimes \mathfrak{A}(\mathfrak{e} / \mathfrak{d}),
$$

where $\mathfrak{A}(\mathfrak{e} / \mathfrak{d})$ denotes the holomorphic functions which are squareintegrable with respect to a canonical Gaussian (see [18]). The point is the action of $N$ in this realization is horrendous, but the description of the action of $H$, i.e. $\tilde{\gamma}$, becomes very simple. This is because we know precisely the weights for the action of $C$ on $\mathfrak{n} / \mathfrak{n}_{\theta}$. In fact we may split $C=B A$ into a direct product of its maximal torus and a split component $A$ so that:

$A$ acts on $\mathfrak{n} / \mathfrak{e}$ by $r=\operatorname{dim} \mathfrak{n} / \mathfrak{e}$ independent positive characters, $A$ acts trivially on $\mathfrak{e} / \mathfrak{d}$,

$B$ acts on $\mathfrak{e} / \mathfrak{d}$ by $s=\operatorname{dim}_{c} \mathfrak{e} / \mathfrak{d}$ independent unitary characters,

$B$ acts trivially on $\mathfrak{n} / \mathfrak{e}$.

Let $K$ be the kernel of $H \rightarrow \mathrm{Sp}\left(\mathfrak{n} / \mathfrak{n}_{\theta}\right)$. Then $H / K \cong C$. It is obvious that $\tilde{\gamma}_{K}=$ Id. Moreover we have (see [15])

$$
\tilde{\gamma}_{H}=2^{r} \cdot \rho_{A} \times \rho_{B}^{+}
$$


where

$$
\begin{gathered}
\text { if } \rho_{B}=\sum_{m \in \mathbb{Z}^{s}} \chi_{m}, \text { then } \rho_{B}^{+}=\sum_{m \in\left(\mathbb{Z}^{s}\right)^{+}} \chi_{m}, \\
\left(\mathbb{Z}^{s}\right)^{+}=\text {the set of } s \text {-tuples of non-negative integers. }
\end{gathered}
$$

This concludes the proof of Lemma 3.3 and Theorem 3.2.

It follows from Theorem 3.2 that the usual Weil representation of $\operatorname{Sp}(n, \mathbb{R})$ is multiplicity-free. Other applications to semisimple groups are possible, but in line with our main interest in algebraic exponential solvable groups, we now specialize $H$ to be a split abelian group.

Proposition 3.4. Let $G=A N$ be algebraic with unipotent radical $N$ and $A$ split abelian. Suppose $\theta \in \mathfrak{n}^{*}, \gamma=\gamma_{\theta} \in \hat{N}$ and $A=A_{\theta}$. If the map $A \rightarrow \mathrm{Sp}\left(\mathfrak{n} / \mathfrak{n}_{\theta}\right)$ is of full rank, then the Weil representation $\tilde{\gamma}$ has uniform multiplicity $2^{r}, r=\frac{1}{2} \operatorname{dim} \mathfrak{n} / \mathfrak{n}_{\theta}$. Otherwise the representation $\tilde{\gamma}$ is of uniform infinite multiplicity. In either case $\tilde{\gamma}$ is quasi-equivalent to the regular representation of $A / A_{0}, A_{0}$ the kernel of $A \rightarrow \operatorname{Sp}\left(\mathfrak{n} / \mathfrak{n}_{\theta}\right)$.

Proof. In either eventuality, we know by the observation in the proof of Theorem 3.2, that the representation $\tilde{\gamma}$ factors to a representation of $A / A_{0}$. The image $C$ of $A$ in $\operatorname{Sp}\left(\mathfrak{n} / \mathfrak{n}_{\theta}\right)$ is a connected subgroup of a split Cartan subgroup, so $C \cong\left(\mathbb{R}^{+}\right)^{s}$. The group $A_{0}$ may act on $\mathfrak{n}_{\theta}$-actually only on $\left.\operatorname{Ker} \theta\right|_{\mathfrak{n}_{\theta}}$-but that is completely irrelevant to the representation $\tilde{\gamma}$. As a representation of $A / A_{0}, \tilde{\gamma}$ is nothing more than the action of $A / A_{0}$ on $L^{2}(\mathfrak{n} / \mathfrak{m})$ given by

$$
\tilde{\gamma}(\alpha) f(x)=\delta(\alpha)^{-1 / 2} f\left(\alpha^{-1} \cdot x\right),
$$

where $A / A_{0}$ acts on $\mathfrak{n} / \mathfrak{m}$ by $s=\operatorname{dim} A / A_{0}$ independent positive characters, $s \leq \frac{1}{2} \operatorname{dim} \mathfrak{n} / \mathfrak{n}_{\theta}, \delta=\delta_{\mathfrak{n} / \mathfrak{m}}$ and $\mathfrak{m}$ is an $A$-invariant real polarization for $\theta$. All of the claims of the proposition are evident from those specifications.

REMARK 3.5. Several people have suggested to me that the full rank situation in Proposition 3.4 is rare-perhaps even restricted to the case that $N$ is Heisenberg. That is not so as the following example reveals. Let $\mathfrak{n}$ be spanned by $X, Y, Z, W$ satisfying $[X, Y]=Z,[X, Z]=W$. Let $A$ be generated by $\exp \mathbb{R} T$ where $T$ acts on $\mathfrak{n}$ by $[T, X]=X,[T, Y]=$ $-2 Y,[T, Z]=-Z,[T, W]=0$. Then for $\theta=W^{*}$, we have $A=A_{\theta}$ and neither $\mathfrak{n}_{\theta}=\mathbb{R} Y+\mathbb{R} W$ nor $\left.\operatorname{ker} \theta\right|_{\mathfrak{n}_{\theta}}=\mathbb{R} Y$ is an ideal in $\mathfrak{n}$. But $A \rightarrow \operatorname{Sp}\left(\mathfrak{n} / \mathfrak{n}_{\theta}\right)$ is of full rank. 
4. Orbital spectrum formula-co-normal case. In this section we prove one of our main results-the orbital spectrum formula for conormal algebraic exponential solvable homogeneous spaces. Let $G=$ $A N$ be a Levi decomposition of the algebraic exponential solvable group $G$ and take $H=A$. Our goal is to prove

THEOREM 4.1. The orbital spectrum formula is true for the conormally induced representation $\operatorname{Ind}_{A}^{A N} 1$, that is

$$
\operatorname{Ind}_{A}^{A N} 1=\int_{\mathfrak{a}^{\perp} / A}^{\oplus} \pi_{\varphi} d \mu(\varphi)
$$

where $\mu$ is the push-forward of Lebesgue measure on $\mathfrak{a}^{\perp}$.

Proof. We employ the strategy of [15]. We prove the result in three stages: (i) $N$ abelian, (ii) $N$ Heisenberg, and then (iii) $N$ arbitrary simply connected nilpotent. In each case $A$ is a simply connected split abelian Lie group, acting semisimply on $\mathfrak{n}$.

(i) $N$ abelian. Then $N$ is just a vector group and $A N / A$ is an abelian symmetric space. The orbital spectrum formula for abelian symmetric spaces was proven in [17, Expl. 8 (iii)].

(ii) $N$ Heisenberg. Let $Z=$ Cent $N$. Consider first the case that $A$ fixes $Z$ pointwise. Then we have a natural map $A \rightarrow \operatorname{Sp}(\mathfrak{n} / \mathfrak{z})$. Let $B$ be the kernel. $B$ is a connected subgroup of $A$ which is central in $G$. Let $C$ be the image of $A$ in $\operatorname{Sp}(\mathfrak{n} / \mathfrak{z}) . C$ is a connected subgroup of a split Cartan subgroup of $\operatorname{Sp}(\mathfrak{n} / \mathfrak{z})$. Set $r=\frac{1}{2} \operatorname{dim} \mathfrak{n} / \mathfrak{z}, s=\operatorname{dim} C, s \leq r$. We have $r>0$ (otherwise $N$ is abelian), and we may take $s>0$ (otherwise $G$ is a direct product $G=A \times N$ and the theorem is evident).

Now we employ the extensive machinery of Theorem 3.1, i.e. the Mackey parameterization of a co-normally induced representation. We write

$$
\pi_{\gamma, \sigma}=\sigma \otimes \tilde{\gamma} \times \gamma, \quad \gamma \in \hat{N} \text { generic (i.e. }\left.\gamma\right|_{z} \neq \text { Id), }
$$

$\tilde{\gamma}$ a Weil representation of $A, \sigma \in \tilde{A}$.

Theorem 3.1 tells us that if

$$
\tilde{\gamma}=\int_{\hat{A}}^{\oplus} n_{\gamma}(\sigma) \sigma d \mu_{\gamma}(\sigma)
$$

then

$$
\operatorname{Ind}_{A}^{A N} 1=\int_{\hat{N}}^{\oplus} \int_{\hat{A}}^{\oplus} n_{\gamma}(\sigma) \pi_{\gamma, \bar{\sigma}} d \mu_{\gamma}(\sigma) d \mu_{N}(\gamma)
$$


Next we avail ourselves of several well-known facts regarding the Weil representations $\tilde{\gamma}$-namely $\tilde{\gamma}(B)=1$ and

$$
\tilde{\gamma}=\omega \rho_{A / B}, \quad \omega= \begin{cases}\infty, & s<r, \\ 2^{r}, & s=r .\end{cases}
$$

Also the measures $\mu_{\gamma}$ are Lebesgue measure.

To prove the equality of formulas (4.1) and (4.2) we must prove equality (a.e.) of the spectra and multiplicities, and equivalence of the measures. We consider the spectra first. It is clear from (4.2)and general principles of the Orbit Method (see [6], [13])-that the representations $\pi_{\gamma, \sigma}$ in (4.2) correspond (generically) to the representations $\pi_{\varphi}, \varphi \in \mathfrak{S}$ where we fix $Z_{0} \in \mathfrak{z}, Z_{0} \neq 0$, choose an $A$-invariant complement $\mathfrak{u}$ to $\mathfrak{z}$, and set

$$
\mathfrak{S}=\left\{\varphi \in \mathfrak{g}^{*}: \theta=\left.\varphi\right|_{\mathfrak{n}}=t Z_{0}^{*}, t \neq 0, \psi=\left.\varphi\right|_{\mathfrak{a}} \in \mathfrak{b}^{\perp}\right\} .
$$

To prove equality of the spectra we must show that (generically) we have

$$
G \cdot \mathfrak{S}=G \cdot \mathfrak{a}^{\perp} \text {. }
$$

First let $\varphi \in \mathfrak{a}^{\perp}$. Set $\theta=\left.\varphi\right|_{\mathfrak{n}}$ and $t=\theta\left(Z_{0}\right)$. Suppose $t \neq 0$ (a generic condition). Choose $n \in N$ such that $n \cdot \theta=t Z_{0}^{*}$ (i.e. $\left.n \cdot \theta(\mathfrak{u})=0\right)$. Then I claim $\left.n \cdot \varphi\right|_{\mathfrak{a}} \in \mathfrak{b}^{\perp}$. In fact if $n=\exp X, X \in \mathfrak{n}$, then for $W \in \mathfrak{b}$

$$
n \cdot \varphi(W)=\varphi(W)-\theta\left([X, W]+\frac{1}{2}[X,[X, W]]-+\cdots\right) \text {. }
$$

The latter is zero because $\varphi(\mathfrak{a})=0$ and $[\mathfrak{b}, \mathfrak{n}]=0$. Hence $n \cdot \varphi \in \mathfrak{S}$. The proof of the reverse inclusion is more difficult. We must take

$$
\varphi=\psi+t Z_{0}^{*}, \quad \psi \in \mathfrak{b}^{\perp} \subset \mathfrak{a}^{*}, \quad t \neq 0,
$$

and show that it can be conjugated into an element of $\mathfrak{a}^{\perp} \subset \mathfrak{g}^{*}$. We diagonalize the action of $A$ on the invariant complement $\mathfrak{u}, \mathfrak{u}=\sum \mathfrak{u}^{\alpha}$

$$
[W, X]=\alpha(W) X, X \in \mathfrak{u}^{\alpha}, \quad W \in \mathfrak{a}, \alpha \in \mathfrak{a}^{*} .
$$

The non-zero weights occur in pairs $\pm \alpha$. Choose an order and let $\alpha_{1}, \ldots, \alpha_{s}$ be the positive weights. Then

$$
\mathfrak{b}=\bigcap_{i=1}^{s} \operatorname{ker} \alpha_{i}
$$

and $\left\{\alpha_{1}, \ldots, \alpha_{s}\right\}$ is a basis of $\mathfrak{b}^{\perp}$. Let $W_{1}, \ldots, W_{s}$ be a dual basis $(\mathfrak{a}=$ $\left.\sum_{i=1}^{s} \mathbb{R} W_{i}+\mathfrak{b}\right)$. We may select $X_{j}, Y_{j} \in \mathfrak{n}$ so that

$$
\begin{aligned}
{\left[W_{i}, X_{j}\right] } & =\delta_{i j} X_{j}, & {\left[W_{i}, Y_{j}\right] } & =-\delta_{i j} Y_{j}, \\
{\left[X_{i}, Y_{j}\right] } & =\delta_{i j} Z_{0} & {\left[X_{i}, X_{j}\right] } & =\left[Y_{i}, Y_{j}\right]=0 .
\end{aligned}
$$


(Note that if $s<r, \sum_{i=1}^{s} R X_{i}+\mathbb{R} Y_{i}+\mathbb{R} Z_{0}$ does not fill up n.) Now if we take an element $n \in N$ of the form

$$
n=\prod_{j} \exp x_{j} X_{j} \exp y_{j} Y_{j}
$$

then it is easy to see that

$$
t Z_{0}^{*}\left(n \cdot W_{i}\right)=t x_{i} y_{i}
$$

Then we may compute

$$
\begin{aligned}
& \varphi(n \cdot W)=0, \quad W \in \mathfrak{b} \quad\left(\text { since }[\mathfrak{b}, \mathfrak{n}]=0, \psi \in \mathfrak{b}^{\perp}\right), \\
& \varphi\left(n \cdot W_{i}\right)=\psi\left(W_{i}\right)+t x_{i} y_{i} .
\end{aligned}
$$

Thus we may select the $x_{i}$ and $y_{i}$ so that

$$
\psi\left(W_{i}\right)+t x_{i} y_{i}=0, \quad i=1,2, \ldots, s .
$$

That is $n^{-1} \cdot \varphi(\mathfrak{a})=0$. This concludes the proof of (4.3).

Next we attend to the multiplicities. We must show that (generically) for $\varphi$ in $G \cdot \mathfrak{S}=G \cdot \mathfrak{a}^{\perp}=N \cdot \mathfrak{a}^{\perp}$ we have

$$
\# A \text { orbits in } G \cdot \varphi \cap \mathfrak{a}^{\perp}= \begin{cases}\infty, & s<r, \\ 2^{r}, & s=r .\end{cases}
$$

When $s<r$ this is easy. We know (by [17]) that the generic dimension of $G \cdot \varphi \cap \mathfrak{a}^{\perp}$ agrees with the generic dimension of the $A$-orbits on $\mathfrak{a}^{\perp} \Leftrightarrow$ the latter is one-half the generic dimension of the $G$-orbits. On $G \cdot \mathfrak{a}^{\perp}=G \cdot \mathfrak{S}$, the generic functionals $\varphi=\psi+t Z_{0}^{*}$ satisfy $\mathfrak{g}_{\varphi}=\mathfrak{a}+\mathfrak{z}$, so the generic dimension of $G \cdot \varphi$ is $2 r$. The generic dimension of the $A$-orbits on $\mathfrak{a}^{\perp}$ is clearly $s$. So we have uniform infinite multiplicity in both (4.1) and (4.2) when $s<r$. To obtain equality when $s=r$ we perform some further computations. Take $\varphi \in \mathfrak{a}^{\perp}$. Write

$$
\begin{aligned}
\varphi & =\sum_{j=1}^{r}\left(\xi_{j} X_{j}^{*}+\eta_{j} Y_{j}^{*}\right)+t Z_{0}^{*}, \\
n & =\prod_{j=1}^{r} \exp y_{j} Y_{j} \exp x_{j} X_{j}
\end{aligned}
$$

One computes that

$$
\begin{aligned}
n^{-1} \cdot \varphi= & \sum_{i=1}^{r}\left(-\xi_{i} x_{i}+\eta_{i} y_{i}+x_{i} y_{i} t\right) W_{i}^{*} \\
& +\left(\xi_{i}-t y_{i}\right) X_{i}^{*}+\left(\eta_{i}+t x_{i}\right) Y_{i}^{*}+t Z_{0}^{*} .
\end{aligned}
$$


Now $A \cdot \varphi \in \mathfrak{a}^{\perp}$ so if $g=a n^{-1}$ then

$$
\begin{aligned}
g \cdot \varphi & \in \mathfrak{a}^{\perp} \Leftrightarrow n^{-1} \cdot \varphi \in \mathfrak{a}^{\perp} \Leftrightarrow \\
& -\xi_{i} x_{i}+\eta_{i} y_{i}+t x_{i} y_{i}=0, \quad i=1,2, \ldots, r .
\end{aligned}
$$

This corroborates that $\operatorname{dim} G \cdot \varphi \cap \mathfrak{a}^{\perp}=r$. But it also gives more precisely that

$$
\begin{aligned}
G \cdot \varphi \cap \mathfrak{a}^{\perp}=\{\tilde{\varphi}= & \sum_{j=1}^{r}\left(\tilde{\xi}_{j} X_{j}^{*}+\tilde{\eta}_{j} Y_{j}^{*}\right) \\
& \left.+t Z_{0}^{*}: \tilde{\xi}_{j} \tilde{\eta}_{j}=\xi_{j} \eta_{j}, j=1, \ldots, r\right\} .
\end{aligned}
$$

Moreover as long as $\Pi_{j} \xi_{j} \eta_{j} \neq 0$ (a generic condition) we have that there are exactly $2^{r}$ orbits, namely

$$
\left\{\tilde{\varphi}: \tilde{\xi}_{j} \tilde{\eta}_{j}=\xi_{j} \eta_{j} \text {, and } \operatorname{sgn}\left(\tilde{\xi}_{j}\right)=\varepsilon_{j}\right\}, \quad \varepsilon_{j}= \pm 1, j=1, \ldots, r .
$$

Thus the multiplicities in (4.1), (4.2) coincide. (Note: An alternate computation may be done by starting with $\varphi=\psi+t Z_{0}^{*}$ and showing that the condition $n \cdot \varphi \in \mathfrak{a}^{\perp}$ amounts to specializing the values $\left.n \cdot \varphi\right|_{W_{j}}$. One again gets $r$ conditions and $2^{r}$ connected hyperboloids. We shall use this type of computation in the general case (iii) below.)

Finally, it behooves me to say something about the measures. In fact, we shall obtain the equivalence of the quotient measures in (4.1) and (4.2) from a very general principle suggested to me by J. Rosenberg. Since it will be useful on other occasions, I separate it out as

Proposition 4.2. Let the Lie group $G$ act smoothly on the $C^{\infty}$ manifold $X$ and suppose $X / G$ is countably separated. Let $X_{1}, X_{2}$ be (locally closed) submanifolds. Let $\mu_{j}$ be a measure on $X_{j}$ in the canonical measure class. Suppose that $G \cdot X_{1}=G \cdot X_{2}$ up to sets of measure zero. Then the push-forwards $\bar{\mu}_{j}$ of $\mu_{j}$ under $X_{j} \rightarrow G \cdot X_{j} / G$ are in the same class.

Proof. Let $d g$ denote Haar measure on $G$. Consider the composite map

$$
G \times X_{j} \rightarrow G \cdot X_{j} \rightarrow G \cdot X_{j} / G, \quad j=1,2 .
$$

The canonical measure class on the first space is $d g \times d \mu_{j}$. The first map is a submersion and so the canonical measure class $d \nu$ on $G \cdot X_{j}$ is the push-forward of $d g \times d \mu_{j}$. But the orbits are locally closed and so $d \bar{\mu}_{j}$ is nothing more than the push-forward of $d \nu_{j}$ under the 
second map. Since $G \cdot X_{1}=G \cdot X_{2}$, the two measure classes of $d \nu_{1}, d \nu_{2}$ coincide. Hence the measures $d \bar{\mu}_{1}$ and $d \bar{\mu}_{2}$ are equivalent.

That the measures in (4.1) and (4.2) are equivalent is a consequence of Proposition 4.2 if we use $\mathfrak{S}$ and $\mathfrak{a}^{\perp}$ for $X_{1}$ and $X_{2}$ inside $\mathfrak{g}^{*}$.

This completes the proof of Theorem 4.1 when $N$ is Heisenberg and $A$ fixes the center of $N$. In fact the argument in this case is typical of several in the rest of the paper. We have given all the details in this case-we will give fewer details thereafter.

Now assume $N$ is Heisenberg, but $A$ doesn't fix $Z=$ Cent $N$ pointwise. Then, since $\operatorname{dim} Z=1$ and $A$ acts semisimply, there are two generic open $A$-orbits in $\hat{N}$. Hence, the generic representations of $G=A N$ are

$$
\pi_{\sigma}^{ \pm}=\operatorname{Ind}_{B N}^{G} \sigma \tilde{\gamma}^{ \pm} \times \gamma^{ \pm}, \quad \sigma \in \hat{B},
$$

where if $A \rightarrow$ Aut $Z_{N}, B$ equals the kernel (a co-dimension one connected subgroup of $A$ ), and $\gamma^{ \pm}$are fixed elements of $\hat{N}$ whose central characters have opposite signs. By Theorem 3.1 we have

$$
\operatorname{Ind}_{A}^{A N} 1=\sum_{ \pm}^{\oplus} \int_{\hat{B}}^{\oplus} n^{ \pm}(\sigma) \pi_{\sigma}^{ \pm} d \mu^{ \pm}(\sigma) \text {, }
$$

where $\mu^{ \pm}$are Lebesgue measures and $n^{ \pm}(\sigma)$ is given as follows. Let $C$ be the kernel of $B \rightarrow \operatorname{Sp}(\mathfrak{n} / \mathfrak{z}), r=\frac{1}{2} \operatorname{dim} \mathfrak{n} / \mathfrak{z}, s=\operatorname{dim} B / C$. Then

$$
n^{ \pm}(\sigma)= \begin{cases}\infty, & s<r \\ 2^{r}, & s=r .\end{cases}
$$

As above we must demonstrate equality of (4.1) and (4.4) in spectra, multiplicity and measure class.

It is clear from (4.4) that the orbital spectrum is generically $G \cdot \mathfrak{S}$ where

$$
\mathfrak{S}=\left\{\varphi \in \mathfrak{g}^{*}: \theta=\left.\varphi\right|_{\mathfrak{n}}=t Z_{0}^{*}, t \neq 0, \psi=\left.\varphi\right|_{\mathfrak{a}} \in \mathfrak{c}^{\perp}\right\} .
$$

So we must show (generically) that

$$
G \cdot \mathfrak{S}=G \cdot \mathfrak{a}^{\perp} .
$$

The inclusion of the right in the left is proven word-for-word as before. The reverse inclusion comes about as follows. Let $\varphi=\psi+t Z_{0}^{*}$. As in the previous situation we find $n \in N$ so that $n \cdot \varphi(\mathfrak{b})=0$. But then we use the fact that for any $\varphi \in \mathfrak{g}^{*},\left.\varphi\right|_{\mathfrak{z}} \neq 0$, if $\theta=\left.\varphi\right|_{\mathfrak{n}}$, then

$$
N_{\theta} \cdot \varphi=\varphi+\left(\mathfrak{g}_{\theta}+\mathfrak{n}\right)^{\perp}=\varphi+(\mathfrak{b}+\mathfrak{n})^{\perp}
$$

(see [1, II.1.3] or [11, p. 271 (iv'] or [20, Lem. 2]). Hence we can further conjugate by $N$ to make the functional vanish on all of $\mathfrak{a}$. 
Regarding multiplicities, the $G$-orbits this time are of dimension $2(r+1)$. The $A$-orbits have dimension $s+1$, and once again all multiplicities are $+\infty$ unless $s=r$. In that case, using the same computation as before (except this time only $\operatorname{sgn}(t)$ is fixed) we get $2^{r}$ hyperbolicshaped $A$-orbits in $G \cdot \varphi \cap \mathfrak{a}^{\perp}$. Finally, an application of Proposition 4.2 gives the equality of the measure classes. This finishes case (ii).

(iii) $N$ arbitrary simply connected nilpotent Lie group. Theorem 3.1 still applies. We have

$$
\operatorname{Ind}_{A}^{A N} 1=\int_{\hat{N} / A}^{\oplus} \int_{\hat{A}_{\gamma}}^{\oplus} n_{\gamma}(\bar{\sigma}) \pi_{\gamma, \sigma} d \sigma d \dot{\mu}_{N}(\gamma),
$$

where $\pi_{\gamma, \sigma}=\operatorname{Ind}_{A_{\gamma} N}^{G} \sigma \otimes \tilde{\gamma} \times \gamma, d \sigma$ is Lebesgue measure and $d \dot{\mu}_{N}$ is the push-forward of Plancherel measure. We can be somewhat more precise. The stability groups $A_{\gamma}$ are connected and reductive. Hence by Theorem 3.2 (i) (the Alignment Lemma), there is $\theta \in \mathscr{O}_{\gamma}$ such that $A_{\gamma}=A_{\theta}$. Then we can describe $\tilde{\gamma}$ virtually as in case (ii). Consider $A_{\theta} \rightarrow \operatorname{Sp}\left(\mathfrak{n} / \mathfrak{n}_{\theta}\right), B_{\theta}$ the kernel, $s=\operatorname{dim} A_{\theta} / B_{\theta}$. We may choose an $A_{\theta}$-invariant polarization $\mathfrak{m}$. The action of $A_{\theta}$ on $\mathfrak{n} / \mathfrak{n}_{\theta}$ generates an action of

$$
\left(\mathbb{R}^{+}\right)^{s}=A_{\theta} / B_{\theta} \quad \text { on } L^{2}\left(\mathbb{R}^{r_{1}}\right), r_{1}=\operatorname{dim} \mathfrak{n} / \mathfrak{m} .
$$

It is quasi-equivalent to the regular representation of $A_{\theta} / B_{\theta}$ and the multiplicity is

$$
\omega= \begin{cases}\infty, & s<r_{1}, \\ 2^{r_{1}}, & s=r_{1} .\end{cases}
$$

The orbital spectrum can be related to the Mackey parameters-namely it is $G \cdot \mathfrak{S}$ where

$$
\mathfrak{S}=\left\{\varphi \in \mathfrak{g}^{*}: \theta=\left.\varphi\right|_{\mathfrak{n}} \text { is aligned, } \psi=\left.\varphi\right|_{\mathfrak{a}} \in \mathfrak{b}_{\theta}^{\perp}\right\} .
$$

So to show equality of spectra in (4.1) and (4.5) we must prove (generically)

$$
G \cdot \mathfrak{S}=G \cdot \mathfrak{a}^{\perp}
$$

In fact, this is a consequence of the following

Proposition 4.3. Let $G=A N$ be algebraic exponential solvable. Let $\varphi \in \mathfrak{a}^{\perp}, \theta=\left.\varphi\right|_{\mathfrak{n}}$. Suppose $A$ fixes $\theta$. Let $B$ be the kernel of the natural map $A \rightarrow \mathrm{Sp}\left(\mathfrak{n} / \mathfrak{n}_{\theta}\right)$. Then $\left.N \cdot \varphi\right|_{\mathfrak{a}}=\mathfrak{b}^{\perp}$ in $\mathfrak{a}^{*}$.

We first explain why the proposition implies equation (4.6). Let $\varphi \in \mathfrak{a}^{\perp}$. Choose $n$ so that $\varphi_{1}=n \cdot \varphi$ is aligned. Write $\varphi_{1}=\psi_{1}+\theta_{1}$. 
Then $\varphi=n^{-1} \cdot \varphi_{1} \in \mathfrak{a}^{\perp}$ says that $\psi_{1}=-\left.n^{-1} \cdot \theta_{1}\right|_{\mathfrak{a}}$. Then apply Proposition 4.3 to get $\psi_{1} \in \mathfrak{b}^{\perp} \subset \mathfrak{a}^{*}$. Conversely, let $\varphi \in \mathfrak{S}$. Write $\varphi=\psi+\theta, \theta$ aligned, $\psi \in \mathfrak{b}^{\perp} \subset \mathfrak{a}^{*}$. Also set $\varphi^{\prime}=0+\theta$. By Proposition 4.3, there is $n$ so that $\left.n \cdot \varphi^{\prime}\right|_{\mathfrak{a}}=-\psi$. That is $\left.n \cdot \varphi\right|_{\mathfrak{a}}=0$. Write $\varphi_{1}=n \cdot \varphi$. Then for $\gamma=\gamma_{\theta}$, we have $\varphi_{1}\left(a_{\gamma}\right)=0$. The functional $\theta_{1}=\left.\varphi_{1}\right|_{\mathfrak{n}}$ may not be aligned. But $\mathfrak{g}_{\gamma_{1}} \cap \mathfrak{a}=\mathfrak{a}_{\gamma_{1}}=\mathfrak{a}_{\gamma}=\mathfrak{a}_{\theta}$. Hence $N_{\theta_{1}} \cdot \varphi_{1}=\varphi_{1}+\left(\mathfrak{a}_{\theta}+\mathfrak{n}\right)^{\perp}$. That is $\varphi_{1}=n \cdot \varphi$ can be further conjugated by an element of $N$ so as to vanish on all of $a$.

Proof of Proposition 4.3. We first show that $\left.N \cdot \theta\right|_{\mathfrak{a}} \subset \mathfrak{b}^{\perp}$. Choose an $A$-invariant complement $\mathfrak{u}$ for $\mathfrak{n}_{\theta}$. Then $N=N_{\theta} \exp \mathfrak{u}$. Let $n \in N$ and write $n=n_{\theta} \exp X, n_{\theta} \in N_{\theta}, X \in \mathfrak{u}$. Then for $W \in \mathfrak{b}, n_{\theta}=\exp Y, Y \in$ $\mathfrak{n}_{\theta}$, we have

$$
\begin{aligned}
\theta(n \cdot W) & =\theta\left(n_{\theta} \exp X \cdot W\right) \\
& =\theta\left(n_{\theta} \cdot W\right) \text { because }[\mathfrak{b}, \mathfrak{u}]=0 \\
& =\theta\left(W-[Y, W]+\frac{1}{2}[Y,[Y, W]]-+\cdots\right)=0,
\end{aligned}
$$

because $\theta(W)=0, W \in \mathfrak{a}=\mathfrak{a}_{\theta}$ and $Y \in \mathfrak{n}_{\theta}$.

Now we must show that $\left.N \cdot \theta\right|_{\mathfrak{a}}$ actually fills up all of $\mathfrak{b}^{\perp}$. The proof of this fact is by induction on $\operatorname{dim} \mathfrak{n}$. The case $\operatorname{dim} \mathfrak{n}=1$ is trivial. So let $G=A N$ and assume the assertion for all groups whose unipotent radical has lower dimension than that of $\mathfrak{n}$. If $N$ is Heisenberg, the result is true by the computation in part (ii), so we may assume $\mathfrak{n}$ is not Heisenberg. Next let $\mathfrak{z}=\operatorname{cent} \mathfrak{n}$ and $\operatorname{set} \mathfrak{z}_{0}=\mathfrak{z} \cap \operatorname{ker} \theta$, an ideal in $\mathfrak{n}$ which is normalized by $A$. The hypotheses and conclusions pass to $\mathfrak{g} / \mathfrak{z}_{0}$. Hence by induction we may assume $\mathfrak{z}_{0}$ is trivial, i.e. $\operatorname{dim} \mathfrak{z}=1$ and $\theta(\mathfrak{z}) \neq 0$. Then we let $\mathfrak{u}=\operatorname{ker} \theta, \mathfrak{n}=\mathfrak{z}+\mathfrak{u}$. Choose any eigenvector $Y \in \mathfrak{z}^{(2)} \cap \mathfrak{u}, \mathfrak{z}^{(2)}$ the second center,

$$
[W, Y]=\alpha(W) Y, \quad W \in \mathfrak{a} .
$$

The usual nilpotent structure theory gives us (see [9]) that the centralizer $\mathfrak{n}_{1}$ of $Y$ in $\mathfrak{n}$ is an $A$-invariant ideal of co-dimension 1 in $\mathfrak{n}$. Furthermore if $\theta_{1}=\left.\theta\right|_{n_{1}}$, then

$$
\mathfrak{n} \supset \mathfrak{n}_{1} \supset\left(\mathfrak{n}_{1}\right)_{\theta_{1}} \supset \mathfrak{n}_{\theta} \quad \text { and } \quad \operatorname{dim} \mathfrak{n} / \mathfrak{n}_{1}=\operatorname{dim}\left(\mathfrak{n}_{1}\right)_{\theta_{1}} / \mathfrak{n}_{\theta}=1 \text {. }
$$

Suppose $A \rightarrow \operatorname{Sp}\left(\mathfrak{n}_{1} /\left(\mathfrak{n}_{1}\right)_{\theta_{1}}\right)$ has the same kernel $B$. (This will occur if $\alpha$ is in the span of the weights for the action of $\mathfrak{a}$ on $\mathfrak{n}_{1} /\left(\mathfrak{n}_{1}\right)_{\theta_{1}}$. $)$ Then by the induction assumption $\left.N_{1} \cdot g_{1}\right|_{\mathfrak{a}}=\mathfrak{b}^{\perp}$ which implies the desired result. 
The other possibility is that the kernel $B_{1}$ of $A \rightarrow \operatorname{Sp}\left(\mathfrak{n}_{1} /\left(\mathfrak{n}_{1}\right)_{\theta_{1}}\right)$ has $\operatorname{dimension}=1+\operatorname{dim} B$. In that case we may write the weights for the action of $\mathfrak{a}$ on $\mathfrak{n} / \mathfrak{n}_{\theta}$ as $\pm \alpha_{1}, \ldots, \alpha_{s}$ and

$$
\mathfrak{b}=\bigcap_{i=1}^{s} \operatorname{ker} \alpha_{i} \quad \mathfrak{b}_{1}=\bigcap_{i=2}^{s} \operatorname{ker} \alpha_{i}
$$

At this point it is no loss of generality to put $\mathfrak{b}=\{0\}$. Then $\operatorname{dim} \mathfrak{b}_{1}=1$. Set $\mathfrak{k}=\operatorname{ker} \alpha_{1}$ so that

$$
\mathfrak{a}=\mathfrak{b}_{1} \oplus \mathfrak{k}
$$

Any $\psi \in \mathfrak{a}^{*}$ can be written uniquely $\psi=\psi_{1}+\psi_{2}, \psi_{1} \in \mathfrak{b}^{\perp}, \psi_{2} \in \mathfrak{k}^{\perp}=$ $\mathbb{R} \alpha_{1}$. Apply the induction hypothesis to conclude:

$$
\exists n_{1} \in N_{1} \text { such that }\left.n_{1} \cdot \theta_{1}\right|_{k}=\psi_{1} \text {. }
$$

Now we may choose an eigenvector $X \in \mathfrak{u} \cap\left(\mathfrak{n} \backslash \mathfrak{n}_{1}\right)$ so that $[X, Y]=$ $Z_{0} \in \mathfrak{z}, \theta\left(Z_{0}\right)=1$,

$$
[W, X]=-\alpha_{1}(W) X, \quad W \in \mathfrak{a} .
$$

There are two possibilities.

(a) $n_{1} \cdot \theta(X)=0$. Then for $x, y \in \mathbb{R}$

$$
\begin{aligned}
n_{1} \cdot \theta & (\exp x X \exp y Y \cdot W) \\
& =n_{1} \cdot \theta\left(W-\alpha_{1}(W) y Y+\alpha_{1}(W) x X-\alpha_{1}(W) x y Z_{0}\right) \\
& =\psi_{1}(W)-\alpha_{1}(W) x y .
\end{aligned}
$$

This shows $\left.N \cdot \theta\right|_{\mathfrak{a}}=\mathfrak{a}^{*}$.

(b) $n_{1} \cdot \theta(X) \neq 0$. Then for $x \in \mathbb{R}$

$$
\begin{aligned}
n_{1} \cdot \theta(\exp x X \cdot W) & =n_{1} \cdot \theta\left(W+\alpha_{1}(W) x X\right) \\
& =\psi_{1}(W)+x\left(n_{1} \cdot \theta\right)(X) \alpha_{1}(W),
\end{aligned}
$$

showing once again that $\left.N \cdot \theta\right|_{\mathfrak{a}}=\mathfrak{a}^{*}$. This completes the proof of Proposition 4.3, and with it the demonstration of equation (4.6).

Next we consider multiplicities. In the aligned format $\varphi=\psi+\theta$, the stabilizers are $\mathfrak{g}_{\varphi}=\mathfrak{a}_{\varphi}+\mathfrak{n}_{\varphi}$ (see [12, Lem. 4.2]). (In that reference, the Levi components are assumed compact, but reductive is all that is necessary.) But $\mathfrak{a}_{\varphi}=\mathfrak{a}_{\theta}$ (since $\mathfrak{a}$ is abelian). Moreover, $N_{\theta} \cdot \varphi=$ $\varphi+\left(\mathfrak{a}_{\theta}+\mathfrak{n}\right)^{\perp}$ says that $\operatorname{dim} \mathfrak{n}_{\theta} / \mathfrak{n}_{\varphi}=\operatorname{dim} \mathfrak{a} / \mathfrak{a}_{\theta}$. Hence the generic dimension of $G$-orbits is $\operatorname{dim} \mathfrak{a} / \mathfrak{a}_{\theta}+\operatorname{dim} \mathfrak{n} / \mathfrak{n}_{\theta}+\operatorname{dim} \mathfrak{n}_{\theta} / \mathfrak{n}_{\varphi}=2\left(r_{1}+r_{2}\right)$ where

$$
\begin{aligned}
& r_{1}=\operatorname{dim} \mathfrak{n} / \mathfrak{m}=\frac{1}{2} \operatorname{dim} \mathfrak{n} / \mathfrak{n}_{\theta}, \quad \mathfrak{m} \text { an } A_{\theta} \text {-invariant polarization for } \theta, \\
& r_{2}=\operatorname{dim} \mathfrak{n}_{\theta} / \mathfrak{n}_{\varphi}=\operatorname{dim} \mathfrak{a} / \mathfrak{a}_{\theta} .
\end{aligned}
$$


Now we must show that (generically) for $\varphi$ in $G \cdot \mathfrak{S}=G \cdot \alpha^{\perp}=N \cdot \mathfrak{a}^{\perp}$ we have

$$
\# A \text {-orbits in } G \cdot \varphi \cap \mathfrak{a}^{\perp}= \begin{cases}\infty, & s<r_{1}, \\ 2^{r_{1}}, & s=r_{1} .\end{cases}
$$

But we can reason as in the Heisenberg case. The generic dimension of the orbit intersections $G \cdot \varphi \cap \mathfrak{a}^{\perp}$ agrees with the generic dimension of $A$-orbits $\Leftrightarrow$ the latter is one-half the generic dimension of the $G$-orbits meeting $\mathfrak{a}^{\perp}$, i.e. $r_{1}+r_{2}$. The generic $A$-orbit obviously has dimension $s+r_{2}$. Hence the equal dimension case occurs precisely when $s=r_{1}$. Thus we have proven equality of multiplicity when $s<r_{1}$, that is uniform infinite multiplicity. So now we may assume $s=r_{1}$. We must show that generically there are $2^{r_{1}} A$-orbits on $G \cdot \varphi \cap \mathfrak{a}^{\perp}$. Now it follows from the duality between $\mathfrak{a} / \mathfrak{a}_{\theta}$ and $\mathfrak{n}_{\theta} / \mathfrak{n}_{\varphi}$ that there is no loss of generality in assuming $\mathfrak{a}=\mathfrak{a}_{\theta}$. The portion of the orbit intersection arising from $A / A_{\theta}$ corresponds to that generated by $N_{\theta} / N_{\varphi}$ and the components are not affected. Moreover, as in the Heisenberg case, we may, without loss of generality, assume $\mathfrak{b}_{\theta}$ is trivial. Hence we are reduced to proving

LEMMA 4.4. Let $G=A N$ be algebraic exponential solvable, $\theta \in \mathfrak{n}^{*}$. Suppose $A=A_{\theta}$ and $A \rightarrow \operatorname{Sp}\left(\mathfrak{n} / \mathfrak{n}_{\theta}\right)$ is a bijection onto a split Cartan (see Rem. 3.5). Let $r=\operatorname{dim} A=\frac{1}{2} \operatorname{dim} \mathfrak{n} / \mathfrak{n}_{\theta}, \varphi \in p_{\mathfrak{g} / \mathfrak{n}}^{-1}(\theta), \psi=\left.\varphi\right|_{\mathfrak{a}}$. Then for generic $\psi$, we have $2^{r}$ A-orbits on $G \cdot \varphi \cap \mathfrak{a}^{\perp}$.

Proof. Let $\mathfrak{n}=\mathfrak{n}_{\theta}+\mathfrak{u}$, an $A$-invariant decomposition. As usual, by induction, we may assume $\operatorname{dim} \mathfrak{z}=1$ and $\theta(\mathfrak{z}) \neq 0$. Then $\mathfrak{n}=\mathfrak{z}+\operatorname{ker} \theta$ is also an $A$-invariant decomposition. Fix $Z_{0} \in \mathfrak{z}$ so that $\theta\left(Z_{0}\right)=1$. The skew form $B_{\theta}(X, Y)=\theta[X, Y]$ is non-degenerate on $\mathfrak{u}$. We diagonalize the action of $\mathfrak{a}$ on $\mathfrak{u}$, namely $\mathfrak{u}=\sum \mathfrak{u}^{\alpha}$ where

$$
[W, X]=\alpha(W) X, \quad X \in \mathfrak{u}^{\alpha}, W \in \mathfrak{a}, \alpha \in \mathfrak{a}^{*} .
$$

The non-zero weights occur in pairs $\pm \alpha$. If we fix an order we have $r$ positive weights $\alpha_{1}, \ldots, \alpha_{r}$ and they are linearly independent. Let $W_{1}, \ldots, W_{r}$ be the dual basis. We select eigenvectors $X_{j}=X_{\alpha_{j}}, Y_{j}=$ $X_{-\alpha_{j}}$, so that

$$
\begin{aligned}
{\left[W_{i}, X_{j}\right] } & =\delta_{i j} X_{j}, \quad\left[W_{i}, Y_{j}\right]=-\delta_{i j} Y_{j}, \\
{\left[X_{i}, Y_{j}\right] } & \equiv \delta_{i j} Z_{0} \quad \bmod \mathfrak{z}, \\
{\left[X_{i}, X_{j}\right] } & \equiv\left[Y_{i}, Y_{j}\right] \equiv 0 \quad \bmod \mathfrak{n}_{\theta} .
\end{aligned}
$$

The generic $\psi$ we have in mind are those for which $\psi_{i}=\psi\left(W_{i}\right) \neq$ $0, i=1,2, \ldots, r$. Then the $A$-orbits on the $G$-orbit intersections with 
$\mathfrak{a}^{\perp}$ are specified by examining the conditions under which the elements

$$
n=\prod_{j} \exp x_{j} X_{j} \exp y_{j} Y_{j}
$$

satisfy $n \cdot \theta=-\psi\left(\right.$ since $\left.N_{\theta} \cdot \varphi=\varphi\right)$. In fact the argument comes down to the

Claim. $n^{-1} \cdot \theta\left(W_{j}\right)=x_{j} y_{j}$.

If this is so, it shows that there are $2^{r}$ hyperbolic components which constitute the $A$-orbits in $G \cdot \varphi \cap \mathfrak{a}^{\perp}$. Now to prove the claim, note that, modulo $\mathfrak{n}_{\theta} \backslash \mathfrak{z}$, we have

$$
n \cdot W_{j}=W_{j}+y_{j} Y_{j}-x_{j} X_{j}+x_{j} y_{j} Z_{0}+\cdots,
$$

where the concluding dots stand for sums of bracket terms of the form

$$
\lambda\left[U_{1},\left[U_{2}, \ldots,\left[U_{k-1}, U_{k}\right] \cdots\right]\right], \quad 2 \leq k \leq r,
$$

in which $\lambda \in \mathbb{R}$ and each $U_{i}$ is one of the eigenvectors. In fact such a bracket must lie in $\mathfrak{n}^{\tilde{\alpha}}, \tilde{\alpha}=\sum \alpha_{j}$ if $U_{j} \in \mathfrak{u}^{\alpha_{j}}$. If $\tilde{\alpha} \neq 0, \theta$ kills the term. If $\tilde{\alpha}=0$ we observe that the only expression with $k=2$ not killed by $\theta$ is accounted for in (4.7). Thus $k \geq 4$ and $k$ must be even. In fact $\theta$ kills those also. For example consider

$$
\left[X_{\alpha},\left[X_{-\alpha},\left[X_{\beta}, X_{-\beta}\right]\right]\right] \text {. }
$$

We have $\left[X_{-\alpha},\left[X_{\beta}, X_{-\beta}\right] \equiv 0 \bmod \mathfrak{n}_{\theta}\right.$, therefore

$$
\theta\left[X_{\alpha}, \mathfrak{n}_{\theta}\right]=0 .
$$

This proves the claim, and so concludes the proof of Lemma 4.4.

Finally we obtain the equivalence of the measure classes from Proposition 4.2 as expected. To apply it we need to observe that $\mathfrak{S}$ is (generically) a manifold. This is true because of [12, Lem. 4.2 (iii)]. If we set $Z_{N}(A)=\left\{n \in N: a n a^{-1}=n, \forall a \in A\right\}$, then $\mathfrak{S}$ is fibered by

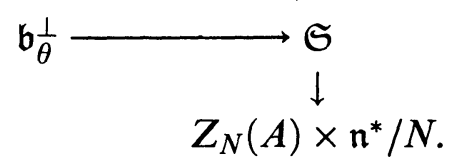

$\mathfrak{n}^{*} / N$ is generically affine, and for each $\theta$ in a cross-section, one obtains all other aligned functionals in the orbit of $\theta$ by forming $Z_{N}(A) \cdot \theta$.

The proof of Theorem 4.1 is now complete. 
5. Orbital spectrum formula-multiplicity-free nilpotent part. We have explained in $\S 2$ (Cor. $2.3 \&$ Lem. 3.4) how, in order to prove the orbital spectrum formula for algebraic exponential solvable homogeneous spaces, we must handle the situation

$$
\operatorname{Ind}_{A M}^{A N} 1 \text {. }
$$

Here $G=A N$ is algebraic exponential solvable, $N$ is the unipotent radical and $A$ is a split Levi component. The algebraic subgroup $H=$ $A M$ has unipotent radical $M \subset N$ and the same Levi component $A$ as $G$. The orbital spectrum formula for $G, H$ asserts that

$$
\operatorname{Ind}_{H}^{G} 1=\int_{(\mathfrak{a}+\mathfrak{m})^{\perp} / A M}^{\oplus} \pi_{\varphi} d \mu(\varphi),
$$

$\mu$ the push-forward of Lebesgue measure. In this section we prove another of our main results - that formula (5.1) is true under the assumption that $\operatorname{Ind}_{M}^{N} 1$ is multiplicity-free. This will be enough to verify the Benoist conjecture $(\S 6)$ (see Rem. 5.4 for a comment on the difficulties that arise in the non-multiplicity-free situation).

We begin by recalling the method of proof of $(5.1)$ in $\S 4$ in the case that $M$ is trivial. The basic requirement is the description of $\operatorname{Ind}_{A}^{A N} 1$ in terms of Mackey parameters. Theorem 3.1 supplies that description. Then the spectrum formula is given by establishing that the Mackey parameters agree with the orbital parameters in the orbital spectrum formula. We follow the same track here, so we need a generalization of Theorem 3.1 to the induced representation $\operatorname{Ind}_{A M}^{A N} 1$. Although we shall only need the case in which $\operatorname{Ind}_{M}^{N} 1$ is multiplicity-free in this paper, we prove the most general possible result. It is our hope that it will prove useful later when the multiplicity-free assumption is removed.

THEOREM 5.1. Let $G=H N$ be a semidirect product with $N$ normal, type I and unimodular. Suppose $M \subset N$ is a closed $H$-invariant subgroup. Let $\hat{N}_{M}$ be the support of $\operatorname{Ind}_{M}^{N}$. Suppose $\hat{N}_{M} / H$ is countably separated. Let

$$
\operatorname{Ind}_{M}^{N} 1=\int_{\hat{N}_{M}}^{\oplus} \gamma d \mu_{M}(\gamma)
$$

be the central decomposition into factor representations. Then for $\mu_{M}-$ a.a. $\gamma$, the space $\mathscr{V}_{\gamma}$ of $\gamma$ is actually an $H_{\gamma} N$-space. Suppose the resulting representations $\nu_{\gamma}$ of $G_{\gamma}=H_{\gamma} N$ are type I for a.a. $\gamma$. Let

$$
\nu_{\gamma}=\int_{\check{G}_{\gamma}}^{\oplus} n_{\gamma}(\nu) \nu d \mu_{\gamma}(\nu)
$$


be their direct integral decompositions. Here $\breve{G}_{\gamma}=\left\{\nu \in \hat{G}_{\gamma}:\left.\nu\right|_{N}\right.$ is quasi-equivalent to $\gamma\}$. Then

$$
\operatorname{Ind}_{H M}^{H N} 1=\int_{\hat{N}_{M} / H}^{\oplus} \int_{\check{G}_{\gamma}}^{\oplus} n_{\gamma}(\nu) \pi_{\nu} d \mu_{\gamma}(\nu) d \dot{\mu}_{M}(\gamma),
$$

where $\pi_{\nu}=\operatorname{Ind}_{G_{y}}^{G} \nu$ and $\dot{\mu}_{M}$ is the push-forward of $\mu_{M}$ to $\hat{N}_{M} / H$.

Proof. The argument is very similar to the proof of Theorem 3.1 that appears in [17]. I shall only give the main points.

First assume that $G$ is any type I group, $\pi$ a unitary representation of $G$,

$$
\pi=\int^{\oplus} \gamma d \mu(\gamma)
$$

the central decomposition of $\pi$, and $\alpha \in \operatorname{Aut}(G)$. Then

$$
\pi \cdot \alpha \cong \int^{\oplus} \gamma \cdot \alpha d \mu(\gamma) \text {. }
$$

(I write group actions on the right in order to correspond to [17].) Suppose that $\pi$ and $\pi \cdot \alpha$ are equivalent. Then $\mu$ is quasi-invariant (under $\alpha$ ) and we may choose unitary maps

$$
\tilde{\gamma}: \mathscr{V}_{\gamma \cdot \alpha} \rightarrow \mathscr{V}_{\gamma}
$$

satisfying

$$
\gamma\left(\alpha^{-1}(g)\right) \tilde{\gamma}_{\alpha}=\tilde{\gamma}_{\alpha}(\gamma \cdot \alpha)(g), \quad g \in G .
$$

The mappings $\tilde{\gamma}_{\alpha}$ are not uniquely specified (even up to scalars) if the factor representations $\gamma$ are not irreducible. However, suppose that a fixed unitary operator $T_{\alpha}$ is chosen effecting the equivalence between $\pi$ and $\pi \cdot \alpha$. Then for $\mu$-a.a. $\gamma$, the "Mackey extensions" $\tilde{\gamma}_{\alpha}$ are uniquely determined by the requirement that the infinitesimal constituents of $T_{\alpha}$ be the maps

$$
\xi_{\gamma} \rightarrow q(\alpha, \gamma)^{1 / 2} \tilde{\gamma}_{\alpha} \xi_{\gamma \cdot \alpha}
$$

$q(\alpha, \gamma)=\left(d \mu \cdot \alpha^{-1}\right) / d \mu$. Moreover the extensions are measurable functions of $\gamma$ and $\alpha$.

Now apply the above generalities to

$$
\pi=\operatorname{Ind}_{M}^{N} 1,
$$

with $H$ a group of automorphisms of $N$ preserving $M$. It is clear that for any $h \in H$,

$$
\pi \cdot h \cong \pi
$$


The quasi-regular representation $\operatorname{Ind}_{M}^{N} 1$ acts on $L^{2}(N ; M)$ (see $\S 1$ for the notation). For $h \in H$, we set

$$
T_{h} f(n)=q_{N}(h)^{-1 / 2} f\left(h^{-1} n h\right), \quad f \in L^{2}(N ; M) .
$$

Then the extension operators $\tilde{\gamma}_{h}$ are uniquely specified. Now the remainder of the argument is almost identical to [17, §7]. The representation $\operatorname{Ind}_{H M}^{H N} 1$ also acts in $L^{2}(N ; M)$. The latter is decomposed over $\hat{N}_{M}$ which we then disintegrate under the action of $H$. Then we let

$$
\Phi_{\gamma}: \mathscr{V}_{\gamma} \rightarrow \int_{\check{G}_{\gamma}}^{\oplus} \mathscr{K}_{\nu} d \mu_{\gamma}(\nu)
$$

be the direct integral decomposition which intertwines $\tilde{\gamma}$ and $\int^{\oplus} n_{\gamma}(\nu) \nu d \mu_{\gamma}(\nu)$. One defines

$$
\Phi: \int_{H / H_{\gamma}}^{\oplus} \mathscr{V}_{\gamma \cdot h} \rightarrow \int_{\check{G}_{\gamma}}^{\oplus} n_{\gamma}(\nu) \mathscr{V}_{\pi_{\nu}} d \mu_{\gamma}(\nu)
$$

by

$$
\Phi:\left\{\xi_{\gamma \cdot h}\right\} \rightarrow\left\{f_{\nu}^{\xi}\right\}
$$

where

$$
f_{\nu}^{\xi}(h)=\Phi_{\gamma}^{\nu} \tilde{\gamma}_{h} \xi_{\gamma \cdot h}
$$

The symbol $\mathscr{V}_{\pi_{\nu}}$ denotes the space of $n_{\gamma}(\nu) \pi_{\nu}=n_{\gamma}(\nu) \operatorname{Ind}_{G_{\gamma}}^{G} \nu$ which is $L^{2}\left(H ; H_{\gamma} ; \mathscr{K}_{\nu}\right)$. Combining the disintegration with the intertwining operator $\Phi$, we obtain the intertwining operator which effects the equivalence in formula (5.2). The corroborating details are virtually identical to $[17, \S 7]$.

Now we put Theorem 5.1 to use in the case that $\operatorname{Ind}_{M}^{N} 1$ is multiplicityfree. Let $G=A N$ be algebraic exponential solvable with algebraic subgroup $H=A M, M \subset N$.

THEOREM 5.2. Suppose $\operatorname{Ind}_{M}^{N} 1$ is multiplicity-free. Then the orbital spectrum formula is valid for the quasi-regular representation $\operatorname{Ind}_{H}^{G} 1$, that is

$$
\operatorname{Ind}_{A M}^{A N} 1=\int_{(\mathfrak{a}+\mathfrak{m})^{\perp} / A M}^{\oplus} \pi_{\varphi} d \mu(\varphi),
$$

$\mu$ the push-forward of Lebesgue measure.

Proof. We begin with the observation that the commuting algebra of the representation $\operatorname{Ind}_{M}^{N} 1$ contains that of $\operatorname{Ind}_{A M}^{A N} 1$. Indeed both representations may be realized in $L^{2}(N ; M)$. Furthermore,

$$
\left.\operatorname{Ind}_{A M}^{A N} 1\right|_{N}=\operatorname{Ind}_{M}^{N} 1 \text {. }
$$


The assertion about commuting algebras follows immediately. Hence the quasi-regular representation $\operatorname{Ind}_{A M}^{A N} 1$ must be multiplicity-free if $\operatorname{Ind}_{M}^{N} 1$ is.

Next we show that the multiplicities in the orbital spectrum formula are generically 1 . That is we prove:

$$
\left.\varphi \in(\mathfrak{a}+\mathfrak{m})^{\perp} \Rightarrow A N \cdot \varphi \cap(\mathfrak{a}+\mathfrak{m})^{\perp}=A M \cdot \varphi \quad \text { (generic } \varphi\right) .
$$

We know that $\theta \in \mathfrak{m}^{\perp} \Rightarrow N \cdot \theta \cap \mathfrak{m}^{\perp}=M \cdot \theta$ is true generically [3]. We shall prove the corresponding result for all $\varphi \in(\mathfrak{a}+\mathfrak{m})^{\perp}$ whose restriction $\theta$ to $\mathfrak{n}$ satisfies $N \cdot \theta \cap \mathfrak{m}^{\perp}=M \cdot \theta$. So suppose $\varphi$ is of this form and

$$
a n \cdot \varphi \in(\mathfrak{a}+\mathfrak{m})^{\perp}, \quad a \in A, n \in N .
$$

Then $n \cdot \theta(\mathfrak{m})=a n \cdot \theta(a \cdot \mathfrak{m})=a n \cdot \varphi(\mathfrak{m})=0$. Hence there exists $m \in M$ such that $n \cdot \theta=m \cdot \theta$. That is $n=m n_{\theta}, n_{\theta} \in N_{\theta}$. Then an $\varphi=a m n_{\theta} \cdot \varphi \in(\mathfrak{a}+\mathfrak{m})^{\perp}$, which implies

$$
\begin{aligned}
n_{\theta} \cdot \varphi(\mathfrak{a}+\mathfrak{m}) & =\operatorname{amn}_{\theta} \cdot \varphi(a m \cdot(\mathfrak{a}+\mathfrak{m})) \\
& =a n \cdot \varphi(\mathfrak{a}+\mathfrak{m})=0 .
\end{aligned}
$$

That is $n_{\theta} \cdot \varphi \in(\mathfrak{a}+\mathfrak{m})^{\perp}$. But $N_{\theta} \cdot \varphi=\varphi+\left(\mathfrak{a}_{\gamma}+\mathfrak{n}\right)^{\perp}$. Thus if $n_{\theta} \cdot \varphi-\varphi$ vanishes on all of $\mathfrak{a}$, it must be that $n_{\theta} \cdot \varphi=\varphi$, i.e. $n_{\theta} \in N_{\varphi}$. Hence $a n \cdot \varphi=a m n_{\theta} \cdot \varphi=a m \cdot \varphi$.

We have demonstrated the multiplicity portion of Theorem 5.2, actually without recourse to Theorem 5.1. We need Theorem 5.1 for the spectrum portion. Indeed in all previous cases (several times in [17] and also in $\S 4$ ), we used Orbit Method procedures to easily read off from the Mackey parameters of a direct integral what the orbital parameters $\mathfrak{S}$ must be. Then we were reduced to showing $G \cdot \mathfrak{S}=G \cdot \mathfrak{h}^{\perp}$. In this case it is not so easy to read off $\mathfrak{S}$. In fact I claim it is

$$
\mathfrak{S}=\left\{\varphi=\xi+\theta: \theta \in \mathfrak{m}^{\perp}, \theta \text { aligned and } \xi\left(\mathfrak{a}_{\theta}\right)=0\right\} .
$$

First we shall prove that $G \cdot \mathfrak{S}=G \cdot(\mathfrak{a}+\mathfrak{m})^{\perp}$, and then we shall show why Theorem 5.1 says the spectrum of $\operatorname{Ind}_{A M}^{A N} 1$ is actually $G \cdot \mathfrak{S}$.

If $\varphi \in \mathfrak{S}$, then $N_{\theta} \cdot \varphi=\varphi+\left(\mathfrak{a}_{\theta}+\mathfrak{n}\right)^{\perp}$. Hence we can conjugate $\varphi$ by an element of $N_{\theta}$ which leaves its restriction to $\mathfrak{n}$ untouched and renders it zero on all of $\mathfrak{a}$. Conversely suppose $\varphi \in(\mathfrak{a}+\mathfrak{m})^{\perp}$. Assuming we can align $\varphi$ by an element $m$ of $M$, it is clear that $m \cdot \varphi \in \mathfrak{S}$. The alignment claim is justified by

LEMMA 5.3 (Refinement of Alignment). Let $G=A N$ be algebraic, $H=A M$ an algebraic subgroup (so $A$ preserves $M$ ). Suppose that 
$\operatorname{Ind}_{M}^{N} 1$ is multiplicity-free, and generically on $\hat{N}_{M}$ the stability group $A_{\gamma}$ is reductive. Then for generic $\gamma \in \hat{N}_{M}$ we may find a functional $\theta$ in the orbit $\mathscr{O}_{\gamma}$ of $\gamma$ which is aligned and in $\mathrm{m}^{\perp}$.

Proof. We consider the (generic) functional $\theta \in \mathfrak{m}^{\perp}$ which satisfies $N \cdot \theta \cap \mathfrak{m}^{\perp}=M \cdot \theta$. By Theorem 3.2 (i) we know there is $n \in N$ such that $n \cdot \theta$ is aligned. That is $G_{\theta}=A_{\theta} N_{\theta}$. The content of this new lemma is that we can select $n$ from $M$. To see this we first note that $H_{\gamma}=A_{\gamma} M$ because $H_{\gamma}=H \cap G_{\gamma}=A M \cap A_{\gamma} N=A_{\gamma} M$. Next we assert that $H_{\gamma}=H_{\theta} M$. In fact if $h \in H_{\gamma}$, then $h \cdot \theta=n \cdot \theta$ for some $n \in N$. But $h \cdot \theta \in \mathfrak{m}^{\perp}$ so $n \cdot \theta=m \cdot \theta$ for some $m \in M$ (by the multiplicity-free assumption). Therefore $h=h m^{-1} m \in H_{\theta} M$. Now we reason as in [12, §4]. We have $A_{\gamma} \cong G_{\gamma} / N \cong G_{\theta} / N_{\theta}$. Choose a Levi component $J, G_{\theta}=J N_{\theta}$. But we also have $A_{\gamma} \cong H_{\gamma} / M \cong H_{\theta} / M_{\theta}$. So the Levi component of $H_{\theta}$ is isomorphic to $J$, and it is no loss of generality to take it to be $J, H_{\theta}=J M_{\theta}$. By the maximal reductivity of a Levi component of $H, H=A M$, there must exist $u \in M$ so that $J \subset u A u^{-1}$. Then exactly as in [12], it is straightforward to verify that $u \cdot \theta$ is aligned.

It remains to show that the spectrum of $\operatorname{Ind}_{A M}^{A N}$ as described by Theorem 5.1 corresponds to the representations $\pi_{\varphi}$ whose orbits $G \cdot \varphi$ meet $\mathfrak{S}$. Now we know that there is a unitary operator

$$
\Psi: L^{2}(N ; M) \rightarrow \int_{\mathfrak{m}^{\perp} / M}^{\oplus} \mathscr{H}_{\theta} d \mu_{M}(\theta)
$$

which commutes

$$
\operatorname{Ind}_{M}^{N} 1 \text { with } \int_{\mathfrak{m}^{\perp} / M}^{\oplus} \gamma_{\theta} d \mu_{M}(\theta) .
$$

Moreover, by the Refinement of Alignment Lemma (Lemma 5.3) we may assume that $\theta \in \mathfrak{m}^{\perp}$ is aligned, i.e. $A_{\gamma_{\theta}}=A_{\theta}$. Furthermore we can realize $\gamma_{\theta}$ by induction via an $A_{\theta}$-invariant polarization $\mathfrak{b}=\mathfrak{b}_{\theta} \subset$ $\mathfrak{n}$ of $\theta$. Now since $L^{2}(N ; M)$ is multiplicity-free, there is a unique representation $\tilde{\gamma}_{\theta}$ of $A_{\theta}$ so that

$$
\operatorname{Ind}_{A M}^{A N} 1=\int_{\mathfrak{m}^{\perp} / A M}^{\oplus} \pi_{\gamma_{\theta}}, \quad \pi_{\gamma_{\theta}}=\operatorname{Ind}_{A_{\theta} N}^{A N} \tilde{\gamma}_{\theta} \times \gamma_{\theta} .
$$

We may realize $\mathscr{H}_{\theta}$ as $L^{2}\left(N ; B ; \chi_{\theta}\right)$ with $N$ acting by right translation. The action of $A$ in $\operatorname{Ind}_{M}^{N} 1$ is by conjugation (with suitable modulus). Its infinitesimal components under $\Psi$ give precisely the actions of $A_{\theta}$ 
via $\tilde{\gamma}_{\theta}$. But the action of $A_{\theta}$ on $\mathscr{H}_{\theta}$ by conjugation satisfies the same equivariance relation with respect to $\gamma_{\theta}$. Hence it must be that

$$
\tilde{\gamma}_{\theta}(a) F(n)=\psi_{\theta}(a) F\left(a^{-1} n a\right) \delta_{N, B}^{-1 / 2}(a), \quad F \in \mathscr{H}_{\theta},
$$

for some unitary character $\psi_{0}$ of $A_{\theta}$. It is clear from the Orbit Method [6], [13] that to prove the orbital parameters of these representations are precisely $\mathfrak{S}$, we must show the

\section{Claim. $\psi_{\theta} \equiv 1$.}

We shall achieve that by invoking Fujiwara's recent derivation of an explicit (that is distribution-theoretic) Plancherel formula for $\operatorname{Ind}_{M}^{N} 1$ (see [7] [8]). We readjust our notation slightly to conform to that of Fujiwara [7], Set:

$$
\begin{aligned}
& \begin{array}{l}
\tau=\operatorname{Ind}_{M}^{N} 1, \\
\alpha=\alpha_{\tau}, \quad \text { the corresponding anti-distribution } \\
\text { (i.e. conjugate linear), }
\end{array} \\
& \alpha: f \rightarrow \overline{f(e)}, \quad f \in \mathscr{H}_{\tau}=L^{2}(N ; M) ; \\
& \gamma_{\theta}=\operatorname{Ind}_{B}^{N} \chi_{\theta}, \\
& \alpha_{\theta} \text { the corresponding anti-distribution, } \\
& \alpha_{\theta}: F \rightarrow \int_{M / M \cap B} \overline{F(m)} d m, \quad F \in \mathscr{H}_{\theta} .
\end{aligned}
$$

It's well known that for any test function $h$, unitary representation $\pi$, and (anti-) distribution $\beta$, the (anti-) distribution $\pi(h) \beta$ is a smooth function. Fujiwara's Plancherel formula is: $\forall h \in \mathscr{D}(N)$

$$
(\tau(h) \alpha, \alpha)=\int_{\mathfrak{m}^{\perp} / M}\left(\gamma_{\theta}(h) \alpha_{\theta}, \alpha_{\theta}\right) d \dot{\theta}
$$

(see [7]). Now if we translate $h$ by an arbitrary group element and throw that term to the other side, we obtain that the infinitesimal components of the distributions $\tau(h) \alpha$ are $\gamma_{\theta}(h) \alpha_{\theta}$. Fujiwara computes these distributions explicitly:

$$
\begin{aligned}
& \tau(h) \alpha=f^{h}, \quad f^{h}(n)=\int_{M} h(n m) d m \quad[7, \text { p. 322], } \\
& \gamma_{\theta}(h) \alpha_{\theta}=\omega_{\theta}^{h}, \quad \omega_{\theta}^{h}(n)=\int_{B / M \cap B} f^{h}\left(n_{\theta} b\right) \chi_{\theta}(b) d b
\end{aligned}
$$

[7, p. 347]. 
But, by the definition of the action of $\tilde{\gamma}_{\theta}$, it must be that the infinitesimal components of $a \cdot f^{h}$ are $\tilde{\gamma}_{\theta}(a) \omega_{\theta}^{h}$. Now we compute

$$
\begin{aligned}
a \cdot f^{h}(n) & =\delta_{N, M}^{-1 / 2}(a) f^{h}\left(a^{-1} n a\right)=\delta_{N, M}^{-1 / 2}(a) \int_{M} h\left(a^{-1} n a m\right) d m \\
& =\delta_{N, M}^{-1 / 2}(a) \delta_{M}^{-1}(a) \int_{M} h\left(a^{-1} n m a\right) d m=\delta_{M}^{-1 / 2}(a) f^{a \cdot h}(n),
\end{aligned}
$$

whose infinitesimal components are

$$
\begin{aligned}
\delta_{M}^{-1 / 2}(a) \int_{B / M \cap B} f^{a \cdot h}(n b) \chi_{\theta}(b) d b \\
=\int_{B / M \cap B} \delta_{N, M}^{-1 / 2}(a) f^{h}\left(a^{-1} n b a\right) \chi_{\theta}(b) d b
\end{aligned}
$$

while

$$
\begin{aligned}
\tilde{\gamma}(a) & \omega_{\theta}^{h}(n)=\psi_{\theta}(a) \delta_{N, B}^{-1 / 2}(a) \omega_{\theta}^{h}\left(a^{-1} n a\right) \\
& =\psi_{0}(a) \delta_{N, B}^{-1 / 2}(a) \int_{B / M \cap B} f^{h}\left(a^{-1} n a b\right) \chi_{\theta}(b) d b \\
& =\psi_{0}(a) \delta_{N, B}^{-1 / 2}(a) \delta_{B / M \cap B}^{-1}(a) \int_{B / M \cap B} f^{h}\left(a^{-1} n b a\right) \chi_{\theta}(b) d b .
\end{aligned}
$$

Thus to prove $\psi_{\theta} \equiv 1$, it suffices to show

$$
\delta_{N, M}^{-1 / 2}=\delta_{N, B}^{-1 / 2} \delta_{B / M \cap B}^{-1}
$$

The latter is

$$
\delta_{N / M \cap B}^{-1 / 2} \delta_{B / M \cap B}^{-1 / 2}=\delta_{N, M}^{-1 / 2} \delta_{M / M \cap B}^{-1 / 2} \delta_{B / M \cap B}^{-1 / 2} .
$$

So it must be shown that

$$
\delta_{M / M \cap B}^{-1 / 2} \delta_{B / M \cap B}^{-1 / 2} \equiv 1 .
$$

But this follows directly and immediately from Fujiwara's expanded form of his Plancherel formula (5.4). If one employs (5.5), formula (5.4) becomes

$$
h_{M}(e)=\int_{\mathfrak{m}^{\perp} / M} \int_{M / M \cap B} \int_{B / M \cap B} h_{M}(m b) \chi_{\theta}(b) d b d m d \theta
$$

where

$$
h_{M}(n)=\int_{M} h(n m) d m .
$$

The equation (5.6) is an easy consequence-it is also proven in $[8, \mathrm{p}$. 34]. 
This concludes the derivation of the spectrum in Theorem 5.2. Finally, the identification of the spectral measure is carried out as usual by means of Proposition 4.2. We simply apply it to the submanifolds $(\mathfrak{a}+\mathfrak{m})^{\perp}$ and $\mathfrak{m}^{\perp}$. (Note $G \cdot \mathfrak{s} / G \approx \mathfrak{m}^{\perp} / M$.)

REMARKS 5.4. (i) When $\operatorname{Ind}_{M}^{N} 1$ is multiplicity-free, Theorem 5.1 says that for a.a. $\gamma \in \hat{N}_{M}$, there is a unique irreducible representation $\nu(\gamma)$ of $A_{\gamma} N$ extending $\gamma$ so that $\operatorname{Ind}_{A M}^{A N} 1=\int_{\hat{N}_{M} / A}^{\oplus} \operatorname{Ind}_{A_{\gamma} N}^{A N} \nu_{\gamma} d \dot{\gamma}$. The content of Theorem 5.2 is that $\nu(\gamma)$ is the canonical Duflo extension [5] [14]. A similar result is established in [16, §4]. It is probably true whenever $A_{\gamma}$ is reductive.

(ii) Theorem 5.2 certainly applies to the case of symmetric spaces. It has been suggested to me that it may not apply to anything else. That is the condition $\operatorname{Ind}_{M}^{N} 1$ multiplicity-free could imply that $N / M$ is symmetric. That is false. The homogeneous space in [3, Expl. 3, p. 60] is not symmetric, but it is multiplicity-free. And it is easy to concoct a one-dimensional split action on it.

(iii) Our hope is to use the techniques developed herein to obtain the orbital spectrum formula for the quasi-regular representation of an arbitrary algebraic exponential solvable homogeneous space. Clearly Corollary 2.3, Lemma 2.4, and Theorem 4.1 and 5.2 go a long way towards that goal. I believe Theorem 5.2 can be extended to the case that $\operatorname{Ind}_{M}^{N} 1$ has finite multiplicity, where Fujiwara's results are still quite explicit. If $\operatorname{Ind}_{M}^{N} 1$ has finite multiplicity (see [3] or [17]), then only finitely many extensions enter into the Plancherel formula of $\operatorname{Ind}_{A M}^{A N} 1$. But it may be difficult to compute the orbital parameters of these extensions - that is to establish a result analogous to the claim in the above proof. Even worse, it is possible to give examples wherein $\operatorname{Ind}_{M}^{N} 1$ has infinite multiplicity, but Ind ${ }_{A M}^{A N} 1$ has either finite or infinite multiplicity. These are the chief impediments to extending Theorem 5.2 to arbitrary algebraic exponential solvable homogeneous spaces. They can be overcome if $N$ is abelian or Heisenberg. But for general $N$ and $M$ (where $L^{2}(N ; M)$ is not multiplicity-free), the computation of the orbital parameters and multiplicities of the extensions $\tilde{\gamma}_{\theta}$, and thus the homogeneous spaces $L^{2}(A N ; A M)$, has not been completed.

6. Benoist's conjecture. Suppose $G / H$ is a symmetric space, meaning here that $G$ is a connected Lie group and $H$ is the stabilizer of an involutive automorphism $\sigma$ of $G$. Suppose $G$ is type $I$. Then it is purely formal that the spectrum of $\operatorname{Ind}_{H}^{G} 1$ is contained in the closed 
set

$$
\hat{G}^{\sigma}=\left\{\pi \in \hat{G}: \pi^{\sigma} \cong \tilde{\pi}\right\} .
$$

By Benoist's conjecture I mean the following (see [2]).

Conjecture 6.1. Suppose $G$ is exponential solvable. Then the support of the spectral measure of $\operatorname{Ind}_{H}^{G} 1$ is precisely $\hat{G}^{\sigma}$.

If $\Omega=\mathscr{O}_{\pi} \subset \mathfrak{g}^{*}$ is the orbit corresponding to $\pi$, then it is easy to see that

$$
\mathscr{O}_{\pi^{\sigma}}=\sigma(\Omega) \text { and } \mathscr{O}_{\bar{\pi}}=-\Omega \text {. }
$$

Moreover $\sigma(\Omega)=-\Omega$ iff $\Omega \cap \mathfrak{h}^{\perp} \neq \varnothing$ [2]. The map $\Omega \rightarrow \pi_{\Omega}$ is continuous. (J. Ludwig has announced that it is open also.) Hence Benoist's conjecture is tantamount to asserting that the spectrum of Ind $_{H}^{G} 1$ is precisely $G \cdot \mathfrak{h}^{\perp}$. If $G$ is nilpotent, this follows from [3] or [17]. Benoist observed as much in [2, §4.3]. But he left the matter unsettled for exponential solvable groups since there was no orbital spectrum formula in that generality.

If one now takes into account the results of $\S 5$, then Conjecture 6.1 is true if $G$ and $H$ are algebraic. Our goal is to show that it is true for arbitrary exponential solvable symmetric spaces.

THEOREM 6.2. Let $G$ be exponential solvable, $\sigma$ an involutive automorphism of $G, H=G^{\sigma}$ the stability group for $\sigma$. Then the quasiregular representation $\operatorname{Ind}_{H}^{G} 1$ obeys the orbital spectrum formula

$$
\operatorname{Ind}_{H}^{G} 1=\int_{\mathfrak{h}^{\perp} / H}^{\oplus} \pi_{\varphi} d \mu(\varphi)
$$

$\mu=$ the push-forward of Lebesgue measure.

Proof. Now exponential solvable symmetric spaces have property (MF), defined in [16]. (Benoist calls it property $\mathscr{P}$.) One of the consequences of property (MF) is that the quasi-regular representation is multiplicity-free [2, Thm. 3.1]. But property (MF) also implies multiplicity one in the orbital spectrum formula. Indeed we have $\mathfrak{g}=\mathfrak{h} \oplus \mathfrak{q}, \mathfrak{q}=$ the -1 eigenspace of (the derivative of) $\sigma$. Also $H \times Q \rightarrow G=H Q, Q=\exp \mathfrak{q}$, is a diffeomorphism of manifolds (see [2] [16]). Hence if $\varphi \in \mathfrak{h}^{\perp} \approx \mathfrak{q}$ and $g \cdot \varphi \in \mathfrak{q}$, then $g=h q \in H Q$ and

$$
\begin{aligned}
-g \cdot \varphi & =\sigma(g \cdot \varphi)=\sigma(h q \cdot \varphi) \\
& =h q^{-1} \cdot(-\varphi) \Rightarrow q^{2} \in G_{\varphi} \Rightarrow q \in G_{\varphi} \Rightarrow g \cdot \varphi=h \cdot \varphi .
\end{aligned}
$$

That is $G \cdot \varphi \cap \mathfrak{h}^{\perp}=H \cdot \varphi$. 
Next we prove $G \cdot \mathfrak{h}^{\perp}$ is the spectrum of $\operatorname{Ind}_{H}^{G} 1$ (the heart of the Benoist conjecture). We obtain it in two stages-first when $G$ and $H$ are algebraic, and then in general. If $G$ and $H$ are algebraic, then by Lemma 2.4 we may assume $H=A M \subset A N=G$. But Theorem 5.2 applies in this event. That is because the unipotent radical is a characteristic subgroup. Hence $\sigma$ preserves $N$ and $N^{\sigma}=N \cap G^{\sigma}=$ $N \cap A M=M$. Therefore $N / M$ is also symmetric.

Now we drop the algebraic assumption. $G$ is any exponential solvable group with involution $\sigma, H=G^{\sigma}$. Let $N$ be the nilradical of $G$, a connected normal subgroup. Consider the normal subgroup $H N$. We have $H \subset H N \triangleleft G$. If $H N$ is a proper subgroup of $G$, then $H$ is subnormal. But in this case subnormal implies strongly subnormal. That is because $G / N$ is a vector group. In any event reasoning exactly as in $\S 2$, we see it is no loss of generality to assume $H N=G$. Next we effect a reduction to a situation which is almost the same as that of algebraic groups.

Consider the exponential solvable group $\tilde{G}$ which is the semidirect product of $H$ and $N$,

$$
\tilde{G}=H \cdot N \text {. }
$$

We have a natural projection $p: \tilde{G} \rightarrow G=H N, p(h, n)=h n$. If we set $M=H \cap N$, the kernel of $p$ is $\left\{\left(m, m^{-1}\right): m \in M\right\}$. Let $\tilde{H}=p^{-1}(H)=$ $\{(h, m): h \in H, m \in M\}$. If we define $\sigma(h, n)=(\sigma h, \sigma n)=(h, \sigma n)$, we obtain an involution of $\tilde{G}$ whose stability subgroup $\tilde{G}^{\sigma}$ is exactly $\tilde{H}$. Now it is a simple exercise to verify the following:

LEMMA 6.3. Suppose $p: G_{1} \rightarrow G_{2}$ is a surjective homomorphism of exponential solvable groups, $H_{j} \subset G_{j}, j=1,2$, closed connected subgroups, $p\left(H_{1}\right)=H_{2}$ and $H_{1} \supset$ ker $p$. If the orbital spectrum formula is valid for $\operatorname{Ind}_{H_{1}}^{G_{1}} 1$, then it is also valid for $\operatorname{Ind}_{H_{2}}^{G_{2}} 1$.

So we are reduced finally to the following situation:

$G=S \cdot N$ semidirect product, $N \triangleleft G, N$ nilpotent

$H=S \cdot M$ semidirect, $M \subset N, L^{2}(N ; M)$ multiplicity-free.

That is, we are in the situation of Theorem 5.2 except that an exponential solvable group $S$ has replaced the split abelian group $A$. But we are also given that $H=G^{\sigma}$ is the stability group of an involution $\sigma$ of $G$. These are precisely the symmetric spaces studied in [16, §4]. Moreover, Conjecture 4.4 of [16] is valid here because for any $\gamma \in \hat{N}$, the stability group $S_{\gamma}$ carries no characters of order 2 . The results of $[16, \S 4]$ and Theorem 5.1 insure that in the Mackey parameters of 
Ind ${ }_{H}^{G} 1$ we have the representations $\pi_{\gamma}=\operatorname{Ind}_{S_{\gamma} N}^{G} \nu(\gamma)$, where $\gamma \in \hat{N}_{M}$ and $\nu(\gamma)=\tilde{\gamma} \times \gamma$ is the canonical Duflo extension of $\gamma$. Therefore the orbital parameters of these representations are those meeting

$$
\mathfrak{S}=\left\{\varphi=\xi+\theta: \theta=\left.\varphi\right|_{\mathfrak{n}} \in \mathfrak{m}^{\perp}, \xi=\left.\varphi\right|_{\mathfrak{s}} \in \mathfrak{s}_{\gamma}^{\perp}\right\} .
$$

The change from $\S 5$ is that since the stability groups $S_{\gamma}$ are no longer reductive, we may not be able to align $\theta$ and keep it in $\mathfrak{m}^{\perp}$. So perhaps $\mathfrak{s}_{\theta} \subsetneq \mathfrak{s}_{\gamma}$. But it doesn't matter here. We still have

$$
G \cdot \mathfrak{S}=G \cdot \mathfrak{h}^{\perp} .
$$

For if $\varphi \in \mathfrak{S}$, then $N_{\theta} \cdot \varphi=\varphi+\left(\mathfrak{s}_{\gamma}+\mathfrak{n}\right)^{\perp}$-hence we can conjugate by an element of $N_{\theta}$ to put $\varphi$ in $\mathfrak{h}^{\perp}=(\mathfrak{s}+\mathfrak{m})^{\perp}$. The reverse inclusion is trivial now since no alignment is required. That is, if $\varphi \in \mathfrak{h}^{\perp}$, then $\theta=\left.\varphi\right|_{\mathfrak{n}} \in \mathfrak{m}^{\perp}$ and $\xi=\left.\varphi\right|_{\mathfrak{s}}=0$. (Conceivably $\left.\varphi\right|_{\mathfrak{g}_{\theta}} \neq 0$, but that is irrelevant.) Hence the spectrum of $\operatorname{Ind}_{H}^{G} 1$ has the correct orbital parameters. The spectral measure is correct also-apply Proposition 4.2 to $\mathfrak{h}^{\perp}$ and $\mathfrak{m}^{\perp}$. (As in Thm. 5.2, we have $G \cdot \mathfrak{S} / G \approx \mathfrak{m}^{\perp} / M$.)

This concludes the proof of Theorem 6.2 and with it the third of our main results - the substantiation of Benoist's conjecture for an arbitrary exponential solvable symmetric space.

\section{REFERENCES}

[1] L. Auslander and B. Kostant, Polarization and unitary representations of solvable Lie groups, Invent. Math., 14 (1971), 255-354.

[2] Y. Benoist, Multiplicité un pour les espaces symétriques exponentiels, Mem. Soc. Math. France, 15 (1984), 1-37.

[3] L. Corwin and F. Greenleaf (with G. Grelaud), Direct integral decompositions and multiplicities for induced representations of nilpotent Lie groups, Trans. Amer. Math. Soc., 304 (1987), 549-583.

[4] L. Corwin and F. Greenleaf, Spectrum and multiplities for restrictions of unitary representations in nilpotent Lie groups, Pacific J. Math., 135 (1988), 233-267.

[5] M. Duflo, Sur les extensions des representations irreductibles des groupes de Lie nilpotents, Ann. Sci. Ecole Norm. Sup., 5 (1972), 71-120.

[6] M. Duflo, Constructions de representations unitaires d'un groupe de Lie, Harmonic Analysis and Group Representations, C.I.M.E., 1982, 129-222; also “Theorie de Mackey pour les groupes de Lie algebres," Acta Math., 149 (1982), 153-213.

[7] H. Fujiwara, Representations monomiales des groupes de Lie nilpotents, Pacific J. Math., 127 (1987), 329-352.

[8] H. Fujiwara and S. Yamagami, Certaines représentations monomiales d'un groupe de Lie resoluble exponentiel, Advanced Studies in Pure Math., Kyoto, (1986), 153-190.

[9] A. Kirillov, Unitary representations of nilpotent Lie groups, Russian Math. Surveys, 17 (1962), 53-104. 
[10] A. Kleppner and R. Lipsman, The Plancherel formula for group extensions, I, Ann. Sci. Ecole Norm. Sup., 5 (1972), 459-515; II, 6 (1973), 103-132.

[11] R. Lipsman, Characters of Lie groups II, J. D'Analyse, 31 (1977), 257-286.

[12] Orbit theory and harmonic analysis on Lie groups with co-compact nilradical, J. Math. Pures. et Appl., 59 (1980), 337-374.

[13] _ Harmonic induction on Lie groups, J. fur die Reine und Ang. Mat., 344 (1983), 120-148.

[14] - On the existence of a generalized Weil representation, Proc. Conf. on Group Reps. (Marseille, 1982), Lecture Notes in Math., vol. 1020, 161-278.

[15] - On the existence of metric polarizations, Rocky Mountain J. Math., 15 (1985), 13-31.

[16] , Harmonic analysis on non-semisimple symmetric spaces, Israel J. Math., 54 (1986), 335-350.

[17] _ Orbital parameters for induced and restricted representations, Trans. Amer. Math. Soc., 313 (1989), 433-473.

[18] C. Moore, Representations of solvable and nilpotent groups and harmonic analysis on nil and solvmanifolds, Proc. Symp. Pure Math., 24 (1973), 3-44.

[19] L. Pukanszky, Unitary representations of solvable Lie groups, Ann. Sci. Ecole Norm. Sup., 4 (1971), 457-608; also Characters of connected Lie groups, Acta Math., 133 (1974), 81-137.

[20] L. Pukanszky, Unitary representations of Lie groups with co-compact radical and applications, Trans. Amer. Math. Soc., 236 (1978), 1-50.

[21] R. Strichartz, Harmonic analysis on Grassmanian bundles, Trans. Amer. Math. Soc., 296 (1986), 387-409.

Received December 29, 1987 and in revised form September 13, 1988. Supported by NSF under DMS87-00551.

UNIVERSITY OF MARYLAND

College Park, MD 20742 



\section{PACIFIC JOURNAL OF MATHEMATICS EDITORS}

\author{
V. S. VARADARAJAN \\ (Managing Editor) \\ University of California \\ Los Angeles, CA 90024-1555-05 \\ Herbert Clemens \\ University of Utah \\ Salt Lake City, UT 84112 \\ THOMAS ENRIGHT \\ University of California, San Diego \\ La Jolla, CA 92093
}

R. FINN

Stanford University

Stanford, CA 94305

HeRMANN FlaschKa

University of Arizona

Tucson, AZ 85721

VAUGHaN F. R. Jones

University of California

Berkeley, CA 94720

STEVEN KeRCKHOFF

Stanford University

Stanford, CA 94305

\author{
ROBION KIRBY \\ University of California \\ Berkeley, CA 94720 \\ C. C. MOORE \\ University of California \\ Berkeley, CA 94720 \\ HAROLD STARK \\ University of California, San Diego \\ La Jolla, CA 92093
}

\section{ASSOCIATE EDITORS}
R. ARENS
E. F. BECKENBACH
B. H. NEUMANN
F. WOLF
K. YoshidA (1906-1982)

\section{SUPPORTING INSTITUTIONS}

UNIVERSITY OF ARIZONA

UNIVERSITY OF BRITISH COLUMBIA

CALIFORNIA INSTITUTE OF TECHNOLOGY

UNIVERSITY OF CALIFORNIA

MONTANA STATE UNIVERSITY

UNIVERSITY OF NEVADA, RENO

NEW MEXICO STATE UNIVERSITY

OREGON STATE UNIVERSITY
UNIVERSITY OF OREGON

UNIVERSITY OF SOUTHERN CALIFORNIA

STANFORD UNIVERSITY

UNIVERSITY OF HAWAII

UNIVERSITY OF TOKYO

UNIVERSITY OF UTAH

WASHINGTON STATE UNIVERSITY

UNIVERSITY OF WASHINGTON 


\section{Pacific Journal of Mathematics}

Vol. 140, No. $1 \quad$ September, 1989

Michel Brestovski, Algebraic independence of solutions of differential

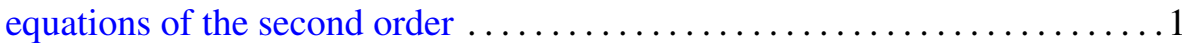

Bohumil Cenkl, Cohomology operations from higher products in the de

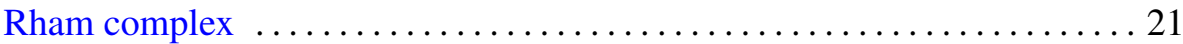

Gustavo Corach and Daniel Suarez, Generalized rational convexity in

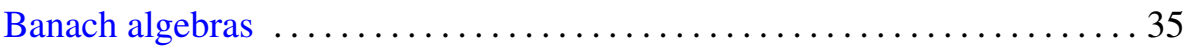

Keresztély Corrádi and Sándor Szabó, A new proof of Rédei’s theorem . . . 53

Steven R. Costenoble and Stefan Waner, Equivariant orientations and

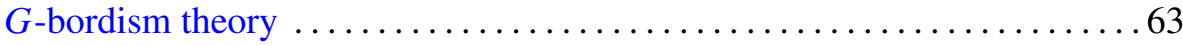

Angel Granja, Apéry basis and polar invariants of plane curve

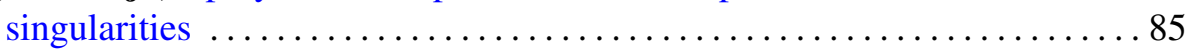

Young Soo Jo, Isometries of tridiagonal algebras .................. 97

Ronald Leslie Lipsman, Harmonic analysis on exponential solvable homogeneous spaces: the algebraic or symmetric cases $\ldots \ldots \ldots \ldots \ldots 117$

Erich Miersemann, On the behaviour of capillaries at a corner 149

Marian Nowak, On the finest Lebesgue topology on the space of essentially

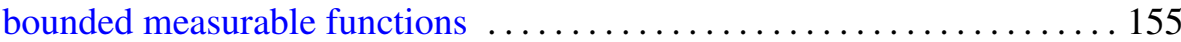

Pascal J. Thomas, Hardy interpolating sequences of hyperplanes ........ 163

H. Bevan Thompson, Differentiability properties of subfunctions for second order ordinary differential equations 